\title{
Weight shiftings for automorphic forms on definite quaternion algebras, and Grothendieck ring
}

\author{
Davide A. Reduzzi
}

\begin{abstract}
Let $p$ be a prime number and let $\mathbb{F}$ be a finite field of characteristic $p$. We investigate the interplay between the algebraic structure of the Grothendieck ring of finitely generated $\mathbb{F}\left[G L_{2}(\mathbb{F})\right]$-modules, and the existence of cohomological operators producing congruences modulo $p$ between automorphic forms on definite quaternion algebras over a totally real field.
\end{abstract}

\section{Introduction}

Let $\mathbb{F}_{q}$ be a finite field of cardinality $q=p^{g}$. In this paper we study the interplay between (1) the existence of some identities in the Grothendieck ring of finitely generated $\mathbb{F}_{q}\left[G L_{2}\left(\mathbb{F}_{q}\right)\right]$-modules, and (2) the existence of congruences modulo $p$ between automorphic forms on definite quaternion algebras. We produce such congruences as maps between cohomology groups. Since our congruences change the weights of the automorphic forms but not the level, they will be called weight shiftings. Our interest on this subject is motivated by the weight part of Serre's modularity conjecture over a totally real field, as formulated by Buzzard-Diamond-Jarvis in [4], and proved by Gee in [10] and [11].

Some of the congruences between Hecke eigensystems that we obtain in the paper are known. The novelty of our results in those cases resides in our method, which is cohomological (as in [3], [8], and [20], which deal with the elliptic case), and in the fact that, via suitable intertwining operators, we can exactly match the existence of weight shiftings between automorphic forms, with the existence of some relations in the Grothendieck ring of $G L_{2}\left(\mathbb{F}_{q}\right)$. We show that these relations (that have the shape of Chebyshev

2010 Mathematics Subject Classification: 11F33, $20 \mathrm{C} 33$.

Key words and phrases: Congruences between automorphic forms, modular representations of $G L_{2}$, Grothendieck ring. 
polynomials) are sufficient to write down a presentation of this ring. (One obtains in this way a heuristic explanation of why some weight shiftings between automorphic forms exist, while others do not: with some exceptions in small weights, only congruences compatible with the structure of the Grothendieck ring are allowed).

Let us first look at the case of elliptic modular forms. For integers $k \geq 2$ and $N \geq 5$, with $N$ prime to $p>3$, denote by $M_{k}\left(N, \overline{\mathbb{F}}_{p}\right)$ the space of elliptic modular forms of weight $k$, level $\Gamma_{1}(N)$, and coefficients in $\overline{\mathbb{F}}_{p}([22]$, $[23])$. The theta operator $\theta: M_{k}\left(N, \overline{\mathbb{F}}_{p}\right) \rightarrow M_{k+(p+1)}\left(N, \overline{\mathbb{F}}_{p}\right)$ is a Hecke twistequivariant map induced on $q$-expansion by the derivation $q d / d q$ and producing a weight shifting of $p+1$. The Hasse invariant $A: M_{k}\left(N, \overline{\mathbb{F}}_{p}\right) \rightarrow$ $M_{k+(p-1)}\left(N, \overline{\mathbb{F}}_{p}\right)$ is a Hecke equivariant map induced by multiplication with the Eisenstein series $E_{p-1}$ and producing a weight shifting of $p-1$. It is known that the Hasse invariant generates all the congruences modulo $p$ between the $q$-expansions of modular forms with $S L_{2}(\mathbb{Z})$ level ([22], 3, Th. 2).

The Eichler-Shimura isomorphism translates the study of Hecke eigensystems of elliptic modular forms over $\overline{\mathbb{F}}_{p}$ of weight $k$ and level $\Gamma_{1}(N)$ into the study of the Hecke action on the group cohomology $H^{1}\left(\Gamma_{1}(N), \operatorname{Sym}^{k-2} \overline{\mathbb{F}}_{p}^{2}\right)$. In [3], Ash and Stevens identify an avatar of the theta operator in the map induced in cohomology by multiplication with the Dickson invariant $\Theta_{p}=X Y^{p}-X^{p} Y$. In [8] Edixhoven and Khare construct a cohomological counterpart of the Hasse invariant acting on weight two forms by studying a degeneracy map

$$
H^{1}\left(\Gamma_{1}(N), \overline{\mathbb{F}}_{p}\right)^{\oplus 2} \rightarrow H^{1}\left(\Gamma_{1}(N) \cap \Gamma_{0}(p), \operatorname{Sym}^{p-1} \overline{\mathbb{F}}_{p}^{2}\right)
$$

A $G L_{2}\left(\mathbb{F}_{p}\right)$-equivariant derivation $D$ of $\mathbb{F}_{p}[X, Y]$ defined by Serre as:

$$
D f=X^{p} \partial_{X} f+Y^{p} \partial_{Y} f
$$

is used in [20] to produce cohomological weight shiftings by $p-1$ starting from forms of weight $2<k \leq p+1$ (Th. 3.4).

The existence of the operators $\Theta_{p}$ and $D$ acting on modular representations of $G L_{2}\left(\mathbb{F}_{p}\right)$ is also suggested by some identities discovered by Serre in the Grothendieck ring $K_{0}\left(G L_{2}\left(\mathbb{F}_{p}\right)\right)$ of finitely generated $\mathbb{F}_{p}\left[G L_{2}\left(\mathbb{F}_{p}\right)\right]$ modules $([25])$. More precisely, set $q=p^{g}$ and for any non-negative integer $k$ define the $G L_{2}\left(\mathbb{F}_{q}\right)$-representation $M_{k}=\operatorname{Sym}^{k} \mathbb{F}_{q}^{2}$. After extending the definition of the $M_{k}$ 's for negative values of $k$ using an Euler characteristic computation (Def. 2.2), Serre observes the following identity between virtual 
representations:

$$
M_{k}-\operatorname{det} \cdot M_{k-(q+1)}=M_{k-(q-1)}-\operatorname{det} \cdot M_{k-2 q}, \quad(k \in \mathbb{Z}) .
$$

When $g=1$, the weight shifting by $p+1$ appearing in (1.0.2) is induced by the Dickson invariant $\Theta_{p}$. Rewriting (1.0.2) as $M_{k}-M_{k-(p-1)}$ $=\operatorname{det} \cdot\left(M_{k-(p+1)}-M_{k-2 p}\right)$, we see that the corresponding weight shifting by $p-1$ is often induced by Serre's derivation $D$. (This breaks down for example when $k=p-1$, as $M_{p-1}$ is irreducible).

It was suggested to us by F. Diamond to look into generalizations of the above results to Hilbert modular forms. Let $F$ be a totally real number field of degree $g>1$ over $\mathbb{Q}$, and let $p$ be a prime unramified in $F$. To simplify the notation of this introduction, we assume that $p$ is inert in $F$, with residue field $\mathbb{F}_{q}$. Working with geometric Hilbert modular forms over $F$ - whose weights correspond to tuples $\mathbf{k}=\left(k_{0}, \ldots, k_{g-1}\right) \in \mathbb{Z}^{g}$ - in [12] Goren defines $g$ partial Hasse invariants, which induce weight shiftings by the vector $(p,-1,0, \ldots, 0)$ and its cyclic permutations. Moreover Katz ([16]) and Andreatta-Goren $([1])$ define $g$ partial theta operators inducing weight shiftings by $(p,+1,0, \ldots, 0)$ and its cyclic permutations.

Motivated by the line of thought described earlier for elliptic modular forms, one can expected to find the following algebraic counterparts of these geometric operators: $(i)$ identities in $K_{0}\left(G L_{2}\left(\mathbb{F}_{q}\right)\right)$ between virtual representations whose weights differ by $(p, \pm 1,0, \ldots, 0) ;(i i)$ intertwining operators between modular representations of $G L_{2}\left(\mathbb{F}_{q}\right)$ producing weight shiftings by $(p, \pm 1,0, \ldots, 0)$.

We discuss $(i)$ in Section 2. In particular, the following relation holds in $K_{0}\left(G L_{2}\left(\mathbb{F}_{q}\right)\right)$ for all integers $h, k$, and $i$ (cf. 2.5):

$$
\begin{aligned}
& M_{k}^{[i]} M_{h}^{[i+1]}-\operatorname{det}^{p^{i+1}} \cdot M_{k-p}^{[i]} M_{h-1}^{[i+1]} \\
= & M_{k-p}^{[i]} M_{h+1}^{[i+1]}-\operatorname{det}^{p^{i+1}} \cdot M_{k-2 p}^{[i]} M_{h}^{[i+1]},
\end{aligned}
$$

where superscripts indicate Frobenius twists. Notice that shiftings by the familiar weights $(p, \pm 1,0, \ldots, 0)$ appear in the above formula. In Section 3 we discuss $(i i)$ and we show that, as for the case $g=1$, the periods appearing in (1.0.3) correspond to weight shiftings arising from intertwining operators. For integers $\alpha, \beta$ such that $0 \leq \alpha \leq g-1$ and $1 \leq \beta \leq g-1$, we define generalized Dickson invariants $\Theta_{\beta}^{[\bar{\alpha}]}$ and generalized $\bar{D}$-operators $D_{\beta}^{[\alpha]}$ giving rise for non-negative integers $k_{0}, \ldots, k_{g-1}$ to the $G L_{2}\left(\mathbb{F}_{q}\right)$-monomorphism:

$$
\Theta_{\beta}^{[\alpha]}: \operatorname{det}^{p^{\alpha}} \otimes \bigotimes_{i} M_{k_{i}}^{[i]} \longrightarrow\left(\bigotimes_{i \neq \alpha, \alpha+\beta} M_{k_{i}}^{[i]}\right) \otimes M_{k_{\alpha}+1}^{[\alpha]} \otimes M_{k_{\alpha+\beta}+p^{g-\beta}}^{[\alpha+\beta]}
$$


and to the $G L_{2}\left(\mathbb{F}_{q}\right)$-morphism:

$$
D_{\beta}^{[\alpha]}: \bigotimes_{i} M_{k_{i}}^{[i]} \longrightarrow\left(\bigotimes_{i \neq \alpha, \alpha+\beta} M_{k_{i}}^{[i]}\right) \otimes M_{k_{\alpha}-1}^{[\alpha]} \otimes M_{k_{\alpha+\beta}+p^{g-\beta}}^{[\alpha+\beta]}
$$

In Section 4 we combine these operators with some results of [6] and [3] on lifting Hecke eigensystems to produce congruences between automorphic forms. Suppose $p$ is an odd prime. Let $\mathcal{O}$ be the ring of integers of the $p$-adic completion of $F$, and let $D$ be a definite quaternion algebra with center $F$ and split at $p$ (recall we are assuming for simplicity that $p$ is inert in $F)$. Denote by $S_{\tau}\left(U, \overline{\mathbb{Z}}_{p}\right)$ the space of $\overline{\mathbb{Z}}_{p}$-valued automorphic forms on $D^{\times}$having prime-to- $p$ level $U \subset\left(D \otimes_{F} \mathbb{A}_{F}^{\infty}\right)^{\times}$and weight $\tau: G L_{2}(\mathcal{O}) \rightarrow$ $\operatorname{Aut}_{\overline{\mathbb{Z}}_{p}}\left(W_{\tau}\right)$ (cf. 4.1). Assume that $\tau$ is regular with parameters $(\mathbf{k}, w)$. We remark that if $U$ is small enough the formation of the spaces $S_{\tau}(U, A)$ for different $\mathcal{O}$-algebras $A$ is compatible with base change (Prop. 4.3 ) - on the other hand, base change compatibility fails for geometric Hilbert modular forms.

An example of weight shifting result obtained using (1.0.4) and (1.0.5) is the following: fix an integer $\beta$ such that $1 \leq \beta \leq g$, and for any $i$ such that $0 \leq i \leq g-1$ choose $a_{i} \in\left\{p^{\beta}-1, p^{\beta}+1\right\}$ and set $\mathbf{a}=\left(a_{0}, \ldots, a_{g-1}\right)$ and $w^{\prime}=w+\left(p^{\beta}-1\right)$.

Theorem (Th. 4.15) Suppose that the weight parameter $(\mathbf{k}, w)$ satisfies the condition $2<k_{i} \leq p+1$ for all $i$, and that $w$ is odd. Then, if $\Omega$ is a Hecke eigensystem occurring in $S_{\tau(\mathbf{k}, w)}\left(U, \overline{\mathbb{Z}}_{p}\right)$, there is a Hecke eigensystem $\Omega^{\prime}$ occurring in regular weight $\left(\mathbf{k}+\mathbf{a}, w^{\prime}\right)$ and level $U$ such that $\Omega^{\prime}\left(\bmod \mathfrak{m}_{\overline{\mathbb{Z}}_{p}}\right)=$ $\Omega\left(\bmod \mathfrak{m}_{\overline{\mathbb{Z}}_{p}}\right)$.

Other weight shiftings can be obtained using various combinations of the operators $\Theta_{\beta}^{[\alpha]}$ and $D_{\beta}^{[\alpha]}$ : for example, shiftings of the form

$$
\left(p^{r}, 0, \ldots, 0, \pm 1,0, \ldots, 0\right)
$$

where the number of zeros between $p^{r}$ and \pm 1 equals $r-1$. In particular, if we set $r=1$ we recover the congruences produced in the geometric context by the partial Hasse and partial theta operators.

As in the elliptic case, the methods of this paper cannot be applied to obtain parallel weight shiftings by $\mathbf{p}-\mathbf{1} \in \mathbb{Z}^{g}$ for forms of weight $\mathbf{k}=\mathbf{2}$, because of the irreducibility of the Steinberg module $M_{q-1}=\bigotimes_{i=0}^{g-1} M_{p-1}^{[i]}$. Using different techniques, Edixhoven and Khare show in [8] that for any non-Eisenstein maximal ideal $\mathfrak{m}$ of the Hecke algebra there is a Heckeequivariant embedding $S_{\mathbf{2}}\left(U, \overline{\mathbb{F}}_{p}\right)_{\mathfrak{m}} \rightarrow S_{\mathbf{p}+\mathbf{1}}\left(U, \overline{\mathbb{F}}_{p}\right)_{\mathfrak{m}}$. Further results on weight shiftings for trivial weights are obtained by C. Sorensen in unpublished work. 
We conclude by observing that identity (1.0.3), whose validity was suggested by the existence of geometric weight shiftings, is sufficient to determine the ring structure of $K_{0}\left(G L_{2}\left(\mathbb{F}_{q}\right)\right)$. This explains the heuristic that the algebraic structure of the Grothendieck ring dictates the existence or non-existence of congruences between automorphic forms.

Using Th. 2.7 we prove in Appendix A the following result, which for simplicity we state for $S L_{2}\left(\mathbb{F}_{q}\right)$-representations:

Theorem (Th. A.6) Denote by $\mathfrak{X}$ the standard representation of $S L_{2}\left(\mathbb{F}_{q}\right)$ on $\mathbb{F}_{q}^{2}$. There is an isomorphism of rings:

$$
K_{0}\left(S L_{2}\left(\mathbb{F}_{q}\right)\right) \simeq \frac{\mathbb{Z}[X]}{(f[g](X)-X)}, \quad \mathfrak{X} \longmapsto X
$$

where $f^{[g]}(X)=(f \circ \cdots \circ f)(X)$ is the $g$-fold self-composition of the monic degree p polynomial:

$$
f(X):=\sum_{j=0}^{\lfloor p / 2\rfloor}(-1)^{j} \frac{p}{p-j}\left(\begin{array}{c}
p-j \\
j
\end{array}\right) X^{p-2 j} .
$$

The polynomial $f(X)$ gives the action of Frobenius on the canonical generator $\mathfrak{X}$ of $K_{0}\left(S L_{2}\left(\mathbb{F}_{q}\right)\right)$. It is interesting to observe that $f(X)$ is a Dickson polynomial of degree $p([5])$, and therefore it is the essentially unique degree $p$ polynomial acting as a "universal Frobenius". More precisely, by a conjecture of Schur $f(X)$ is - up to composition with rational linear polynomials - the only integral degree $p$ polynomial inducing a permutation on infinitely many prime fields (Rmk. A.7).

In recent years there has been considerable focus on the study of weights in Serre-type conjectures for reductive groups $\mathbf{G}$ which are not forms of $G L_{2}$ (cf. work of Herzig [13], Herzig-Tilouine [14], Emerton-Gee-Herzig [7], et.al.). We hope in future work to apply the ideas of this paper to such groups, exploiting the structure of the Grothendieck ring of $\mathbf{G}$ to produce congruences between automorphic forms.

\section{Relations in the Grothendieck ring}

Fix a rational prime $p$, a positive integer $g$, and set $q=p^{g}$. Denote by $\mathbb{F}_{q}$ a finite field with $q$ elements and fix an algebraic closure $\overline{\mathbb{F}}_{q}$ of $\mathbb{F}_{q}$; denote by $\sigma$ the arithmetic Frobenius automorphism of $\mathbb{F}_{q}$. Let $G$ denote the group 
$G L_{2}\left(\mathbb{F}_{q}\right)$ and let $M$ be a representation of $G$ over $\mathbb{F}_{q}$. If $n$ is any integer, $\sigma^{n}$ induces a map $G \rightarrow G$. Composing this map with the action of $G$ on $M$ gives the latter a new structure of $G$-module, denoted $M^{[n]}$ and called the $n$th Frobenius twist of $M$. If $f: M \rightarrow N$ is a $G$-homomorphism and $n \in \mathbb{Z}$, denote by $f^{[n]}: M^{[n]} \rightarrow N^{[n]}$ the induced $G$-homomorphism.

Let $M_{1}$ denote the standard representation of $G$ on $\mathbb{F}_{q}^{2}$ and for any positive integer $k$ define $M_{k}=\operatorname{Sym}^{k} M_{1}$. We identify $M_{k}$ with the $\mathbb{F}_{q}$-vector space of homogeneous polynomials over $\mathbb{F}_{q}$ in two variables $X, Y$ and of degree $k$, endowed with the action of $G$ induced by:

$$
\left(\begin{array}{ll}
a & b \\
c & d
\end{array}\right) \cdot X=a X+c Y,\left(\begin{array}{ll}
a & b \\
c & d
\end{array}\right) \cdot Y=b X+d Y .
$$

We set $M_{0}$ to be the trivial representation of $G$. Denote by det : $G \rightarrow \mathbb{F}_{q}^{\times}$ the determinant character of $G$.

The irreducible representations of $G$ over $\mathbb{F}_{q}$ are all and only of the form ([26], §13):

$$
\operatorname{det}^{m} \otimes \bigotimes_{i=0}^{g-1} M_{k_{i}}^{[i]}
$$

where $k_{0}, \ldots, k_{g-1}$ and $m$ are integers such that $0 \leq k_{i} \leq p-1$ and $0 \leq m<q-1$. The tensor products in (2.0.7) are over $\mathbb{F}_{q}$, and the above representations are pairwise non-isomorphic.

We denote by $K_{0}(G)$ the Grothendieck group of finitely generated $\mathbb{F}_{q}[G]$ modules: it is the free abelian group generated by the isomorphism classes of irreducible representations of $G$ over $\mathbb{F}_{q}([24])$. If $M$ is an $\mathbb{F}_{q}[G]$-module, we also denote by $M$ its class in $K_{0}(G)$. Moreover, we denote by $e$ the image of det in $K_{0}(G)$. Tensor product over $\mathbb{F}_{q}$ induces on $K_{0}(G)$ a structure of commutative ring with identity. We denote the product in the Grothendieck ring $K_{0}(G)$ by · or by juxtaposition.

In this section we present some identities between virtual representations of $G$.

\subsection{Negative weights}

First, we extend the definition of $M_{k} \in K_{0}(G)$ for $k<0$ in a way that is compatible with Brauer character computations, as done by Serre in [25].

Let $\mathbf{G}=G L_{2}$ as an algebraic group over $\mathbb{F}_{q}$, and let $\mathbf{T} \subset \mathbf{G}$ be the maximal torus of diagonal matrices. Identify the character group $X(\mathbf{T})$ of $\mathbf{T}$ with $\mathbb{Z}^{2}$ in the usual way, so that the roots associated to $(\mathbf{G}, \mathbf{T})$ are $(1,-1)$ and 
$(-1,1)$; fix a choice of positive root $\alpha=(1,-1)$. The corresponding Borel subgroup $\mathbf{B}$ is the group of upper triangular matrices in $\mathbf{G}$; we denote by $\mathbf{B}^{-}$the opposite Borel subgroup. For a fixed $\lambda \in X(\mathbf{T})$, let $\mathbf{M}_{\lambda}$ be the one dimensional left $\mathbf{B}^{-}$-module on which $\mathbf{B}^{-}$acts (through $\mathbf{T}$ ) via the character $\lambda$. Denote by ind $\mathbf{B}^{-} \mathbf{M}_{\lambda}$ the left $\mathbf{G}$-module given by algebraic induction from $\mathbf{B}^{-}$to $\mathbf{G}$ of $\mathbf{M}_{\lambda}$. Define the following generalized dual Weyl module for $\lambda$ (cf. [15], II.5):

$$
W(\lambda):=\sum_{i \geq 0}(-1)^{i} \cdot R^{i} \operatorname{ind}_{\mathbf{B}^{-}}^{\mathbf{G}}\left(\mathbf{M}_{\lambda}\right) .
$$

Observe that $W(\lambda)$ is an element of the Grothendieck group $K_{0}(\mathbf{G})$ of $\mathbf{G}$, and that $R^{i}$ ind $_{\mathbf{B}^{-}}^{\mathbf{G}}\left(\mathbf{M}_{\lambda}\right)$ is zero for $i>1$ ([15], II.4.2). For $\lambda_{k}=(k, 0) \in$ $X(\mathbf{T})$ with $k$ any integer we have:

$$
R^{i} \operatorname{ind}_{\mathbf{B}^{-}}^{\mathbf{G}}\left(\mathbf{M}_{\lambda_{k}}\right) \simeq H^{i}\left(\mathbb{P}_{\mathbb{F}_{q}}^{1}, \mathcal{O}(k)\right)
$$

If $k \geq 0$, then $W\left(\lambda_{k}\right)=M_{k}$ (with abuse of notation, as $W\left(\lambda_{k}\right)$ is an algebraic representation of $\mathbf{G}$, while $M_{k}$ is a representation of the specialization $\left.\mathbf{G}\left(\mathbb{F}_{q}\right)\right)$; if $k<0$ we have $W\left(\lambda_{k}\right)=-H^{1}\left(\mathbb{P}_{\mathbb{F}_{q}}^{1}, \mathcal{O}(k)\right)$. The canonical perfect pairing of $\mathbf{G}$-modules:

$$
H^{0}\left(\mathbb{P}_{\mathbb{F}_{q}}^{1}, \mathcal{O}(-k-2)\right) \times H^{1}\left(\mathbb{P}_{\mathbb{F}_{q}}^{1}, \mathcal{O}(k)\right) \rightarrow H^{1}\left(\mathbb{P}_{\mathbb{F}_{q}}^{1}, \mathcal{O}(-2)\right) \simeq \operatorname{det}^{-1} \otimes \mathbf{G}_{a, \mathbb{F}_{q}},
$$

leads to the following:

Definition 2.2 (Serre). Let $k<0$ be an integer. Define the virtual representation $M_{k} \in K_{0}(G)$ by:

$$
M_{k}:=\left\{\begin{array}{cc}
0 & \text { if } k=-1 \\
-e^{1+k} \cdot M_{-k-2} & \text { if } k \leq-2 .
\end{array}\right.
$$

Tautologically, for any $k \in \mathbb{Z}$ we have in $K_{0}(G)$ the identity:

$$
M_{k}+e^{1+k} \cdot M_{-k-2}=0
$$

By slightly abuse of language, we call the integer $k$ attached to $M_{k}$ the weight of the virtual representation.

\subsection{Serre's identity}

Fix an embedding $\iota: \mathbb{F}_{q^{2}} \rightarrow M_{2}\left(\mathbb{F}_{q}\right)$. Let $\overline{\mathbb{Q}}_{p}$ be an algebraic closure of the $p$ adic field $\mathbb{Q}_{p}$ and fix an isomorphism between $\overline{\mathbb{F}}_{q}$ and the residue field of the 
ring of integers $\overline{\mathbb{Z}}_{p}$ of $\overline{\mathbb{Q}}_{p}$. Denote the corresponding Teichmüller character by $\sim: \overline{\mathbb{F}}_{q}^{\times} \rightarrow \overline{\mathbb{Z}}_{p}^{\times}$. Let $a, b \in \mathbb{F}_{q}^{\times}$with $a \neq b$, and let $c \in \mathbb{F}_{q^{2}}^{\times} \backslash \mathbb{F}_{q}^{\times}$. For any $k \geq 1$ the Brauer character $G_{\text {reg }} \rightarrow \overline{\mathbb{Q}}_{p}$ of the representation $M_{k}$ is given by:

$$
\begin{aligned}
\left(\begin{array}{cc}
a & \\
& a
\end{array}\right) & \mapsto(k+1) \cdot \tilde{a}^{k} \\
\left(\begin{array}{cc}
a & \\
& b
\end{array}\right) & \mapsto \frac{\tilde{a}^{k+1}-\tilde{b}^{k+1}}{\tilde{a}-\tilde{b}} \\
\iota(c) & \mapsto \frac{\tilde{c}^{q(k+1)}-\tilde{c}^{k+1}}{\tilde{c}^{q}-\tilde{c}} .
\end{aligned}
$$

Using the above formulae, in [25] Serre observes the following identity, valid in $K_{0}(G)$ for any weight $k \in \mathbb{Z}$ :

$$
M_{k}-e \cdot M_{k-(q+1)}=M_{k-(q-1)}-e \cdot M_{k-2 q} \cdot
$$

\subsection{Product formula}

A computation with Brauer characters also shows that for any $n, m \in \mathbb{Z}$ we have in $K_{0}(G)$ :

$$
M_{n} M_{m}=M_{n+m}+e M_{n-1} M_{m-1}
$$

\subsection{Frobenius twists}

When $g=1$, identities $\left(\Delta_{1}\right)$ and $\left(\Sigma_{1}\right)$ are sufficient to compute the JordanHölder factors of any virtual representation $M_{k}(k \in \mathbb{Z})$. When $g>1$ this is not true, as the above identities do not involve Frobenius twists. We have the following:

Lemma 2.6. Let $g \geq 1$. For any $k \in \mathbb{Z}$ we have in $K_{0}(G)$ the identity:

$$
M_{k}=M_{k-p} M_{1}^{[1]}-e^{p} M_{k-2 p}
$$

Proof. Fix an embedding $\iota: \mathbb{F}_{q^{2}} \rightarrow M_{2}\left(\mathbb{F}_{q}\right)$ and denote by $\tilde{x} \in \overline{\mathbb{Z}}_{p}$ the Teichmüller lift of $x \in \overline{\mathbb{F}}_{q}^{\times}$as in 2.3. Let $\tau$ be the Brauer character of the virtual representation $M_{k}-M_{k-p} M_{1}^{[1]}+e^{p} M_{k-2 p}$. Let $a, b \in \mathbb{F}_{q}^{\times}$such that $a \neq b$. 
We have:

$$
\begin{aligned}
\tau\left(\begin{array}{cc}
a & \\
& a
\end{array}\right)= & (k+1) \tilde{a}^{k}-(k-p+1) \tilde{a}^{k-p} \cdot 2 \tilde{a}^{p} \\
& +\tilde{a}^{2 p} \cdot(k-2 p+1) \tilde{a}^{k-2 p} ; \\
\tau\left(\begin{array}{cc}
a & \\
& b
\end{array}\right)= & \frac{\tilde{a}^{k+1}-\tilde{b}^{k+1}}{\tilde{a}-\tilde{b}}-\frac{\tilde{a}^{k-p+1}-\tilde{b}^{k-p+1}}{\tilde{a}-\tilde{b}} \cdot \frac{\tilde{a}^{2 p}-\tilde{b}^{2 p}}{\tilde{a}^{p}-\tilde{b}^{p}} \\
& +\tilde{a}^{p} \tilde{b}^{p} \cdot \frac{\tilde{a}^{k-2 p+1}-\tilde{b}^{k-2 p+1}}{\tilde{a}-\tilde{b}} .
\end{aligned}
$$

Both these expressions are zero. If $c \in \mathbb{F}_{q^{2}}^{\times} \backslash \mathbb{F}_{q}^{\times}$then $\operatorname{det} \iota(c)=c^{1+q}$; also notice that $\operatorname{tr}\left(\iota(c) ; M_{1}^{[1]}\right)=\operatorname{tr}\left(\iota(c)^{\sigma} ; M_{1}\right)=c^{p}+c^{p q}$, so that:

$$
\begin{aligned}
\tau(\iota(c))= & \frac{\tilde{c}^{q(k+1)}-\tilde{c}^{k+1}}{\tilde{c}^{q}-\tilde{c}}-\left(\tilde{c}^{p}+\tilde{c}^{p q}\right) \cdot \frac{\tilde{c}^{q(k-p+1)}-\tilde{c}^{k-p+1}}{\tilde{c}^{q}-\tilde{c}} \\
& +\tilde{c}^{(1+q) p} \cdot \frac{\tilde{c}^{q(k-2 p+1)}-\tilde{c}^{k-2 p+1}}{\tilde{c}^{q}-\tilde{c}}
\end{aligned}
$$

and this is also zero.

Multiplying $\left(\Phi_{g}\right)$ by $M_{h}^{[1]}$ and applying $\left(\Pi_{g}\right)$ we deduce the following formula, valid for any $k, h, i \in \mathbb{Z}$ :

$$
\left(\Phi_{g}^{\prime}\right) \quad M_{k}^{[i]} M_{h}^{[i+1]}-e^{p^{i+1}} M_{k-p}^{[i]} M_{h-1}^{[i+1]}=M_{k-p}^{[i]} M_{h+1}^{[i+1]}-e^{p^{i+1}} M_{k-2 p}^{[i]} M_{h}^{[i+1]} .
$$

We summarize the identities described above:

Theorem 2.7. Let $q=p^{g}$ and let $k, n, m \in \mathbb{Z}$. The following identities hold in the ring $K_{0}(G)$ :

$\left(\Delta_{g}\right)$

$$
\begin{aligned}
M_{k} & =-e^{1+k} \cdot M_{-k-2} \\
M_{k}-e \cdot M_{k-(q+1)} & =M_{k-(q-1)}-e \cdot M_{k-2 q} \\
M_{n} M_{m} & =M_{n+m}+e M_{n-1} M_{m-1} . \\
M_{k} & =M_{k-p} M_{1}^{[1]}-e^{p} M_{k-2 p} .
\end{aligned}
$$

Remark 2.8. Equations $\left(\Delta_{g}\right),\left(\Pi_{g}\right)$, and $\left(\Phi_{g}\right)$ are sufficient to compute the Jordan-Hölder constituents of virtual representations of the form $\prod_{i=0}^{g-1} M_{k_{i}}^{[i]}$, for all $k_{i} \in \mathbb{Z}$. In particular, Serre's relation $\left(\Sigma_{g}\right)$ is a consequence of the relations $\left(\Delta_{g}\right),\left(\Phi_{g}\right)$, and $\left(\Pi_{g}\right)$. In the appendix, we use Th. 2.7 to obtain 
an explicit presentation for the Grothendieck ring of finite dimensional $\mathbb{F}_{q}\left[S L_{2}\left(\mathbb{F}_{q}\right)\right]$-modules (Th. A.6).

\section{Intertwining operators}

In this section we first recall some intertwining operators between $G$-modules considered in [3], [8], and [20]. Motivated by relation $\left(\Phi_{g}^{\prime}\right)$ of 2.5 and by [12] and [1], we then define in 3.5 generalizations of such operators, which we apply in Section 4 to produce congruences between automorphic forms.

\subsection{The periods $q+1$ and $q-1$}

The irreducible complex representations of $G=G L_{2}\left(\mathbb{F}_{q}\right)$ that are not onedimensional or twists of the Steinberg representation are of two types: the principal series representations, of dimension $q+1$ and obtained by inducing to $G$ regular characters of a Borel subgroup, and the cuspidal representations, of dimension $q-1$ and characterized by the property that they do not occur as factors of a principal series. The two periods $q+1$ and $q-1$ appear in Serre's relation $\left(\Sigma_{g}\right)$ of Th. 2.7. On one hand, this suggests the existence of intertwining operators between $\mathbb{F}_{q}[G]$-modules that shift weights by $q+1$ and $q-1$ respectively. On the other hand, Khare observed that this also suggests the existence of natural $p$-adic integral models of principal series and cuspidal representations of $G$, whose reductions $(\bmod p)$ should coincide with the cokernels of the above intertwining operators. These facts are discussed in [3] and [20], and we recall below the available results.

3.1.1. The period $\boldsymbol{q}+\mathbf{1}$. Let $k>q$ be an integer and let $\Theta_{q}:=X Y^{q}-$ $X^{q} Y \in \mathbb{F}_{q}[X, Y]$ be one of the Dickson invariants for the action of $S L_{2}\left(\mathbb{F}_{q}\right)$ on the symmetric algebra Sym* $\mathbb{F}_{q}^{2}$. Let us denote by $\Theta_{q}$ also the $G$-equivariant map det $\otimes M_{k-(q+1)} \rightarrow M_{k}$ obtained by multiplication by $\Theta_{q}$. There is a natural isomorphism of $G$-modules (cf. [3], Lemma 3.2):

$$
\operatorname{coker} \Theta_{q \mid \operatorname{det} \otimes M_{k-(q+1)}} \stackrel{\simeq}{\longrightarrow} \operatorname{Ind}_{B}^{G}\left(\eta^{k}\right),
$$

where $\eta$ is the character of the upper Borel subgroup $B$ of $G$, defined extending the character $\operatorname{diag}(a, b) \mapsto a$ of the standard maximal torus of $G$.

3.1.2. The period $q-1$. The period $q-1$ is studied in [20]. The starting point is the $G$-equivariant derivation map $D: \mathbb{F}_{q}[X, Y] \rightarrow \mathbb{F}_{q}[X, Y]$ defined 
by Serre as:

$$
D: \quad f(X, Y) \longmapsto X^{q} \frac{\partial f}{\partial X}+Y^{q} \frac{\partial f}{\partial Y} .
$$

By restriction $D$ induces an intertwining operator $M_{k} \rightarrow M_{k+(q-1)}$ for any $k \geq 0$, giving rise to a weight shifting by $q-1$. The kernel of $D$ on $M_{k}$ is often non trivial ([20], Prop. 3.3), and $D$ captures essential properties related to the existence or non-existence of embeddings of $G$-modules of the form $M_{k} \rightarrow M_{k+(q-1)}$ ([20], Prop. 3.5 and Prop. 3.6).

Assume that $p$ is an odd prime and denote by $W\left(\mathbb{F}_{q}\right)$ the ring of Witt vectors for the field $\mathbb{F}_{q}$. Fix an embedding of $W\left(\mathbb{F}_{q}\right)$ inside $\overline{\mathbb{Q}}_{p}$.

Theorem 3.2. Let $k$ be an integer satisfying $2 \leq k \leq p-1$ and $k \neq \frac{q+1}{2}$. Denote by $R(k)$ the cuspidal $\overline{\mathbb{Q}}_{p}$-representation of $G$ associated to the $k$ thpower of the Teichmüller character. Let $C$ be the Deligne-Lusztig variety of $S L_{2 / \mathbb{F}_{q}} \cdot$ There exists a canonical $W\left(\mathbb{F}_{q}\right)$-integral model

$$
\tilde{R}(k):=H_{\text {cris }}^{1}\left(C_{/ \mathbb{F}_{q}}\right)_{-k}
$$

of $R(k)$, arising from the $(-k)$-eigenspace of the first crystalline cohomology group of $C_{/ \mathbb{F}_{q}}$, such that there is a canonical isomorphism of $\mathbb{F}_{q}[G]$-modules:

$$
\operatorname{coker} D_{\mid M_{k}} \simeq \tilde{R}(k) \otimes_{W\left(\mathbb{F}_{q}\right)} \mathbb{F}_{q} \text {. }
$$

The $(-k)$-eigenspace of $H_{\mathrm{cris}}^{1}\left(C_{/ \mathbb{F}_{q}}\right)$ is taken with respect to the natural action of $\operatorname{ker}\left(\mathrm{Nm}_{\mathbb{F}_{q^{2}}^{\times} / \mathbb{F}_{q}^{\times}}\right)$.

Proof. [20], Th. 4.2.

\subsection{Application to elliptic modular forms}

We assume $p>3$ and by a modular form $(\bmod p)$ in this paragraph we mean the reduction modulo $p$ of a form in characteristic zero, as considered by Serre $([23])$ and Swinnerton-Dyer $([22])$. In this paragraph we assume $g=1$, so that $G=G L_{2}\left(\mathbb{F}_{p}\right)$.

Let $N \geq 5$ be a positive integer not divisible by $p$ and denote by $M_{k}\left(N, \overline{\mathbb{F}}_{p}\right)$ the $\overline{\mathbb{F}}_{p}$-vector space of modular forms for the group $\Gamma_{1}(N)$ having weight $k \geq 2$ and with coefficients in $\overline{\mathbb{F}}_{p}$. The Hecke algebra $\mathbb{T}$, generated over $\overline{\mathbb{F}}_{p}$ by the Hecke operators $T_{l}$ for $l \nmid p N$, acts on this space.

The theta operator $\theta: M_{k}\left(N, \overline{\mathbb{F}}_{p}\right) \rightarrow M_{k+(p+1)}\left(N, \overline{\mathbb{F}}_{p}\right)$ is defined on $q$ expansion by the formula $\theta\left(\sum_{n} a_{n} q^{n}\right):=\sum_{n} n a_{n} q^{n}$. If $f \in M_{k}\left(N, \overline{\mathbb{F}}_{p}\right)$ we have $\theta T_{l}(f)=l \cdot T_{l} \theta(f)$ for any $T_{l} \in \mathbb{T}$ with $l \nmid p N$. 
Denote by $E_{p-1}$ the normalized form of the classical Eisenstein series whose $q$-expansion is given by: $E_{p-1}=1-\frac{2(p-1)}{B_{p-1}} \sum_{n \geq 1} \sigma_{p-2}(n) q^{n}$, where $B_{p-1}$ is a Bernoulli number, and $\sigma_{p-2}$ is the divisor sum function. Then $E_{p-1}$ is a modular form of weight $p-1$, level 1 and coefficients in $\mathbb{Z}_{(p)}$. Moreover the $q$-expansion of $E_{p-1}$ is congruent to 1 modulo $p$. Multiplication by $E_{p-1}$ gives rise to a Hecke-equivariant injective map $M_{k}\left(N, \overline{\mathbb{F}}_{p}\right) \rightarrow$ $M_{k+(p-1)}\left(N, \overline{\mathbb{F}}_{p}\right)$, which is multiplication by the Hasse invariant.

In view of the Eichler-Shimura isomorphism, the study of Hecke eigensystems of $(\bmod p)$ modular forms of weight $k \geq 2$ and level $N$ leads to the study of the eigenvalues of the Hecke algebra acting on the cohomology group

$$
H^{1}\left(\Gamma_{1}(N), M_{k-2}\right)
$$

where $\Gamma_{1}(N)$ acts on the $G$-representation $M_{k-2}=\operatorname{Sym}^{k-2} \mathbb{F}_{p}^{2}$ via its reduction $(\bmod p)$, and the action of $\mathbb{T}$ comes from the $G$-action on $M_{k-2}$ as in $[3]$.

The weight shiftings realized on the spaces of modular forms by the theta operator and the Hasse invariant have cohomological counterparts. In [3], Ash and Stevens identifies a group-theoretical analogue of the theta operator in the map induced in cohomology by the Dickson invariant discussed in 3.1.1:

$$
\Theta_{p, *}: H^{1}\left(\Gamma_{1}(N), \operatorname{det} \otimes M_{k-2}\right) \longrightarrow H^{1}\left(\Gamma_{1}(N), M_{k+p-1}\right) .
$$

Edixhoven and Khare identifies in [8] a cohomological analogue of the Hasse invariant in the case $k=2$ by studying the degeneracy map

$$
H^{1}\left(\Gamma_{1}(N), M_{0}\right)^{\oplus 2} \rightarrow H^{1}\left(\Gamma_{1}(N) \cap \Gamma_{0}(p), M_{p-1}\right)
$$

In [20], Serre's derivation map $D$ defined in 3.1.2 is used to allow weight shiftings by $p-1$ when $2<k \leq p+1$ :

Theorem 3.4. Let $\mathfrak{m}$ be a non-Eisenstein maximal ideal of the Hecke algebra $\mathbb{T}$.

1) If $k \geq 0$ and $H^{1}\left(\Gamma_{1}(N), M_{k}\right)_{\mathfrak{m}} \neq 0$, then also $H^{1}\left(\Gamma_{1}(N), M_{k+(p-1)}\right)_{\mathfrak{m}} \neq$ 0 .

2) If $0 \leq k \leq p-1$, there is a Hecke-equivariant embedding

$$
H^{1}\left(\Gamma_{1}(N), M_{k}\right)_{\mathfrak{m}} \hookrightarrow H^{1}\left(\Gamma_{1}(N), M_{k+(p-1)}\right)_{\mathfrak{m}}
$$


that is induced by the derivation $D$ if $0<k \leq p-1$, and is the map defined in [8] if $k=0$.

Proof. The first statement and the second statement for $k \neq 0$ are proved in [20], Prop. 5.1; the second statement in the case $k=0$ is proved in [8], 2.

\subsection{More intertwining operators}

Recall that $G=G L_{2}\left(\mathbb{F}_{q}\right)$ with $q=p^{g}$. When $g=2$, equation $\left(\Phi_{2}^{\prime}\right)$ of 2.5 suggests the existence of intertwining operators shifting weights by $(p, \pm 1)$ and $( \pm 1, p)$. This is also compatible with the weight shiftings produced by partial Hasse invariants and partial theta operators acting on spaces of Hilbert modular forms for a quadratic totally real field (cf. [12], [1]). In this paragraph we define the expected generalizations of $\Theta_{q}$ and $D$. Unless otherwise specified we assume $g>1$. Moreover, all the tensor products considered below are over $\mathbb{F}_{q}$.

3.5.1. Generalized Dickson invariants. For integers $\alpha, \beta$ such that $0 \leq$ $\alpha \leq g-1$ and $1 \leq \beta \leq g-1$, define the generalized Dickson invariant $\Theta_{\beta}^{[\bar{\alpha}]}$ to be the element

$$
\Theta_{\beta}^{[\alpha]}:=X \otimes Y^{p^{g-\beta}}-Y \otimes X^{p^{g-\beta}}
$$

of the $G$-module $M_{1}^{[\alpha]} \otimes M_{p^{g-\beta}}^{[\alpha+\beta]}$. Set moreover $\Theta_{\beta}:=\Theta_{\beta}^{[0]}$. If $k, h$ are nonnegative integers, multiplication by $\Theta_{\beta}^{[\alpha]}$ in the $\mathbb{F}_{q}[G]$-algebra $\mathbb{F}_{q}[X, Y]^{[\alpha]} \otimes$ $\mathbb{F}_{q}[X, Y]^{[\alpha+\beta]}$ induces an injective $G$-homomorphism:

$$
\Theta_{\beta}^{[\alpha]}: \operatorname{det}^{p^{\alpha}} \otimes M_{k}^{[\alpha]} \otimes M_{h}^{[\alpha+\beta]} \rightarrow M_{k+1}^{[\alpha]} \otimes M_{h+p^{g-\beta}}^{[\alpha+\beta]} .
$$

Moreover the classical Dickson invariant $\Theta^{[\alpha]}=X Y^{q}-Y X^{q}$ of 3.1.1, viewed as an element of $M_{q+1}^{[\alpha]}$, induces an injective $G$-homomorphism $\Theta^{[\alpha]}$ : $\operatorname{det}^{p^{\alpha}} \otimes M_{k}^{[\alpha]} \rightarrow M_{k+(q+1)}^{[\alpha]}$.

Remark 3.6. When $\beta=g-1$, the operators $\Theta_{g-1}^{[\alpha]}$ give, under suitable assumptions (cf. Section 4), cohomological analogues of the partial theta operators defined in [1] in the context of Hilbert modular forms. Moreover, the weight shifting by $(\ldots, p, 1, \ldots)$ appearing on both sides of the identity $\left(\Phi_{g}^{\prime}\right)$ of Section 2.5 is realized by $\Theta_{g-1}^{[i+1]}$ acting on modular representations of $G$. 
When $g>2$ the generalized Dickson invariants do not produce weight shiftings by cyclic permutations of $(1, p, 0, \ldots, 0)$. The following computation shows that this is a consequence of the structure of $K_{0}(G)$ :

Proposition 3.7. Assume $g>2$ and let $k, h$ be integers such that $0 \leq$ $k, h \leq p-1$. For any integer $\alpha$ such that $0 \leq \alpha \leq g-1$ and any integer $m$, there are no non-zero G-module morphisms det $^{m} \otimes M_{k}^{[\alpha]} \otimes M_{h}^{[\alpha+1]} \rightarrow$ $M_{k+1}^{[\alpha]} \otimes M_{h+p}^{[\alpha+1]}$.

Proof. Assume $\alpha=0$. By $\left(\Phi_{g}\right)$ and $\left(\Delta_{g}\right)$ we have $M_{h+p}^{[1]}=M_{h}^{[1]} M_{1}^{[2]}+$ $e^{p(h+1)} M_{p-h-2}^{[1]}$. If $k \neq p-1$ we deduce that the Jordan-Hölder factors of $M_{k+1} \otimes M_{h+p}^{[1]}$ are $M_{k+1} \otimes M_{h}^{[1]} \otimes M_{1}^{[2]}$ and $\operatorname{det}^{p(h+1)} \otimes M_{k+1} \otimes M_{p-h-2}^{[1]}$, unless $h=p-1$, in which case only the first factor occurs. This proves the result for $k \neq p-1$, as the Jordan-Hölder factors of $\operatorname{det}^{m} \otimes M_{k} \otimes M_{h}^{[1]}$ and $M_{k+1} \otimes M_{h+p}^{[1]}$ are pairwise non-isomorphic.

Assume now $k=p-1$ and write $M_{p}=M_{1}^{[1]}+e M_{p-2}$. Applying $\left(\Pi_{g}\right)$ we obtain:

$$
\begin{aligned}
M_{p} M_{h+p}^{[1]} & =\left(M_{1}^{[1]}+e M_{p-2}\right)\left(M_{h}^{[1]} M_{1}^{[2]}+e^{p(h+1)} M_{p-h-2}^{[1]}\right) \\
& =M_{h+1}^{[1]} M_{1}^{[2]}+e^{p} M_{h-1}^{[1]} M_{1}^{[2]}+e^{p(h+1)} M_{p-h-1}^{[1]}+e^{p(h+2)} M_{p-h-3}^{[1]} \\
& +e M_{p-2} M_{h}^{[1]} M_{1}^{[2]}+e^{p(h+1)+1} M_{p-2} M_{p-h-2}^{[1]} .
\end{aligned}
$$

If $h \neq p-1$, the above formula shows that none of the Jordan-Hölder factors of $M_{p} \otimes M_{h+p}^{[1]}$ equals $\operatorname{det}^{m} \otimes M_{p-1} \otimes M_{h}^{[1]}$. If $h=p-1$, we have:

$$
\begin{aligned}
M_{p} M_{2 p-1}^{[1]} & =M_{1}^{[2]} M_{1}^{[2]}+e^{p} M_{p-2}^{[1]} M_{1}^{[2]}+e^{p} M_{p-2}^{[1]} M_{1}^{[2]}+e M_{p-2} M_{p-1}^{[1]} M_{1}^{[2]} \\
& =M_{2}^{[2]}+e^{p^{2}}+2 e^{p} M_{p-2}^{[1]} M_{1}^{[2]}+e M_{p-2} M_{p-1}^{[1]} M_{1}^{[2]}
\end{aligned}
$$

and $\operatorname{det}^{m} \otimes M_{p-1} \otimes M_{p-1}^{[1]}$ is not a constituent of $M_{p} \otimes M_{2 p-1}^{[1]}$ if $p \neq 2$. If $p=2$, decomposing further $M_{2}^{[2]}$ we reach the same conclusion.

3.7.1. Generalized $\boldsymbol{D}$-operators. Denote by $\partial_{X}\left(\operatorname{resp} . \partial_{Y}\right)$ the operator of partial derivation with respect to $X$ (resp. $Y$ ) acting on $\mathbb{F}_{q}[X, Y]$. If $f \in$ $\mathbb{F}_{q}[X, Y]$, denote by the same symbol the endomorphism of $\mathbb{F}_{q}[X, Y]$ induced by multiplication by $f$. Let $k, h$ be two non-negative integers; for any integers 
$\alpha, \beta$ such that $0 \leq \alpha \leq g-1$ and $1 \leq \beta \leq g-1$, define the generalized $D$ operator $D_{\beta}^{[\alpha]}$ by:

$$
D_{\beta}^{[\alpha]}:=\partial_{X} \otimes X^{p^{g-\beta}}+\partial_{Y} \otimes Y^{p^{g-\beta}}: M_{k}^{[\alpha]} \otimes M_{h}^{[\alpha+\beta]} \longrightarrow M_{k-1}^{[\alpha]} \otimes M_{h+p^{g-\beta}}^{[\alpha+\beta]}
$$

We set $D_{\beta}:=D_{\beta}^{[0]}$. It is easily checked that $D_{\beta}^{[\alpha]}: M_{k}^{[\alpha]} \otimes M_{h}^{[\alpha+\beta]} \longrightarrow M_{k-1}^{[\alpha]} \otimes$ $M_{h+p^{g-\beta}}^{[\alpha+\beta]}$ is a $G$-homomorphism, and it is injective if $0<k \leq p-1$ and $0 \leq h \leq p-1$. For $k$ and $\alpha$ as above, the Frobenius twists of Serre's operator $D^{[\alpha]}=X^{q} \partial_{X}+Y^{q} \partial_{Y}$ also define $G$-homomorphisms $D^{[\alpha]}: M_{k}^{[\alpha]} \rightarrow M_{k+(q-1)}^{[\alpha]}$ which are injective if $1 \leq k \leq p-1$.

Remark 3.8. When $\beta=g-1$, the operators $D_{g-1}^{[\alpha]}$ for $0 \leq \alpha \leq g-1$ give rise to cohomological analogues of the partial Hasse invariants defined in [1] for Hilbert modular forms (cf. 4). Moreover, rewriting the identity $\left(\Phi_{g}^{\prime}\right)$ of Section 2.5 as:

$$
M_{k}^{[i]} M_{h}^{[i+1]}-M_{k-p}^{[i]} M_{h+1}^{[i+1]}=e^{p^{i+1}} \cdot\left(M_{k-p}^{[i]} M_{h-1}^{[i+1]}-M_{k-2 p}^{[i]} M_{h}^{[i+1]}\right)
$$

we see that the weight shifting by $(\ldots, p,-1, \ldots)$ appearing above is sometimes realized by $D_{g-1}^{[i+1]}$ acting on modular representations of $G$.

Similarly to Prop. 3.7, the nonexistence of weight shiftings by a cyclic permutation of $(-1, p, 0, \ldots, 0)$ when $g>2$ is a consequence of the structure of $K_{0}(G)$.

\section{Congruences between automorphic forms}

We apply the constructions of the previous section to obtain, via cohomological methods, weight shiftings for automorphic forms on definite quaternion algebras.

We begin by fixing our notation. Let $F$ be a totally real number field of degree $g$ over $\mathbb{Q}$, and let $p>2$ be a rational prime unramified in $F$. Denote by $\mathcal{O}_{F}$ the ring of integers of $F$ and write $p \mathcal{O}_{F}=\prod_{j=1}^{r} \mathfrak{P}_{j}$, where the $\mathfrak{P}_{j}$ 's are maximal ideals of $\mathcal{O}_{F}$. Fix an integer $j$ with $1 \leq j \leq r$. Suppose that $\mathbb{F}_{\mathfrak{P}_{j}}:=\mathcal{O}_{F} / \mathfrak{P}_{j}$ is an extension of $\mathbb{F}_{p}=\mathbb{Z} / p \mathbb{Z}$ of degree $f_{j}$. Let $F_{\mathfrak{P}_{j}}$ be the completion of $F$ at $\mathfrak{P}_{j}$, and denote by $\mathcal{O}_{F_{\mathfrak{P}_{j}}}$ its ring of integers. Fix an algebraic closure $\overline{\mathbb{Q}}_{p}$ of $\mathbb{Q}_{p}$; let $n$ be the positive least common multiple of the integers $f_{1}, \ldots, f_{r}$ and let $E$ be the maximal unramified extension of $\mathbb{Q}_{p}$ inside $\overline{\mathbb{Q}}_{p}$ having degree $n$ over $\mathbb{Q}_{p}$, so that $\operatorname{Hom}\left(F, \overline{\mathbb{Q}}_{p}\right)=\operatorname{Hom}(F, E)$. 
Denote by $\mathcal{O}$ the ring of integers of $E$ and let $\mathbb{F}$ be its residue field. Let $\sigma$ be the arithmetic Frobenius of the extension $E / \mathbb{Q}_{p}$. Set:

$$
\operatorname{Hom}\left(F_{\mathfrak{P}_{j}}, E\right)=\left\{\sigma_{i}^{(j)}: 0 \leq i \leq f_{j}-1\right\}
$$

where the labeling is chosen so that for any $i$ we have $\sigma \circ \sigma_{i}^{(j)}=\sigma_{i+1}^{(j)}$; here the subscripts are taken modulo $f_{j}$. Denote by a bar the analogous morphisms for the residue fields, so that $\bar{\sigma}$ is the arithmetic Frobenius of the extension $\mathbb{F} / \mathbb{F}_{p}$, and the elements of $\operatorname{Hom}\left(\mathbb{F}_{\mathfrak{P}_{j}}, \mathbb{F}\right)=\left\{\bar{\sigma}_{i}^{(j)}: 0 \leq i \leq f_{j}-1\right\}$ are labeled so that $\bar{\sigma} \circ \bar{\sigma}_{i}^{(j)}=\bar{\sigma}_{i+1}^{(j)}$.

We let $\mathbb{A}_{F}$ be the ring of adèles of $F$, and we denote by $\mathbb{A}_{F}^{\infty}$ the subring of finite adèles. We let $S_{f}$ (resp. $S_{\infty}$ ) be the set of finite (resp. infinite) places of $F$ and we identify $S_{f}$ with the set of maximal ideals of $\mathcal{O}_{F}$.

\subsection{Automorphic forms on definite quaternion algebras}

We recall basic facts on automorphic forms on definite quaternion algebras over totally real number fields, following [28] and [17]; cf. also [27].

Fix a finite set $\Sigma \subset S_{f}$ disjoint from the set of places of $F$ lying above $p$ and such that $\# \Sigma+[F: \mathbb{Q}]$ is even. Let $D$ be a quaternion algebra over $F$ whose ramification set is $S_{\infty} \cup \Sigma$. Let $\mathcal{O}_{D}$ be a fixed maximal order of $D$ and for any $v \in S_{f}-\Sigma$ fix ring isomorphisms $\left(\mathcal{O}_{D}\right)_{v} \simeq M_{2}\left(\mathcal{O}_{F_{v}}\right)$. Let $U$ be a compact open subgroup of $\left(D \otimes_{F} \mathbb{A}_{F}^{\infty}\right)^{\times}$such that:

1) $U=\prod_{v \in S_{f}} U_{v}$, where $U_{v}$ is a subgroup of $\left(\mathcal{O}_{D}\right)_{v}^{\times}$;

2) $U_{v}=\left(\mathcal{O}_{D}\right)_{v}^{\times}$if $v \in \Sigma$;

3) if $v \mid p$, then $U_{v}=G L_{2}\left(\mathcal{O}_{F_{v}}\right)$.

Let $A$ be a topological $\mathbb{Z}_{p}$-algebra. Let $v_{j}:=\mathfrak{P}_{j}$ be a place of $F$ above $p$, and let $W_{\tau_{j}}$ be a free $A$-module of finite rank. Fix a continuous homomorphism $\tau_{j}: U_{v_{j}}=G L_{2}\left(\mathcal{O}_{F_{\mathfrak{P}_{j}}}\right) \rightarrow \operatorname{Aut}\left(W_{\tau_{j}}\right)$, where $\operatorname{Aut}\left(W_{\tau_{j}}\right)$ is the group of continuous $A$-linear automorphisms of $W_{\tau_{j}}$. Let $W_{\tau}=\bigotimes_{j=1}^{r} W_{\tau_{j}}$, where the tensor products are over $A$, and denote by $\tau$ the corresponding homomorphism $\tau: \prod_{j=1}^{r} U_{v_{j}} \rightarrow \operatorname{Aut}\left(W_{\tau}\right)$. We also denote by $\tau$ the action of $U$ on $W_{\tau}$ induced by the projection $U \rightarrow \prod_{j=1}^{r} U_{v_{j}}$. (We remark that all the $A$-modules we consider are of finite type, so we do not encounter issues on the topology of tensor products, cf. Prop. 4.3) 
For $A$ as above, let $\psi:\left(\mathbb{A}_{F}^{\infty}\right)^{\times} / F^{\times} \rightarrow A^{\times}$be a continuous character such that, for any $v \in S_{f}$ :

$$
\tau_{\mid U_{v} \cap \mathcal{O}_{F_{v}}^{\times}}(u)=\psi^{-1}(u) \cdot I d_{W_{\tau}}, \quad \text { for all } u \in U_{v} \cap \mathcal{O}_{F_{v}}^{\times}
$$

We say that such a Hecke character $\psi$ is compatible with $\tau$.

Definition 4.2. For $D, U, A, \tau, W_{\tau}$ and $\psi$ as above, the space $S_{\tau, \psi}(U, A)$ of automorphic forms on $D^{\times}$having level $U$, weight $\tau$, character $\psi$ and coefficients in $A$ is the $A$-module consisting of functions $f: D^{\times} \backslash\left(D \otimes_{F} \mathbb{A}_{F}^{\infty}\right)^{\times} \longrightarrow$ $W_{\tau}$ satisfying:

1) $f(g u)=\tau(u)^{-1} f(g)$ for all $g \in\left(D \otimes_{F} \mathbb{A}_{F}^{\infty}\right)^{\times}$and all $u \in U$;

2) $f(g z)=\psi(z) f(g)$ for all $g \in\left(D \otimes_{F} \mathbb{A}_{F}^{\infty}\right)^{\times}$and all $z \in\left(\mathbb{A}_{F}^{\infty}\right)^{\times}$.

We assume that for all $t \in\left(D \otimes_{F} \mathbb{A}_{F}^{\infty}\right)^{\times}$, the finite group $\left(U \cdot\left(\mathbb{A}_{F}^{\infty}\right)^{\times} \cap\right.$ $\left.t^{-1} D^{\times} t\right) / F^{\times}$has order prime to $p$. This assumption is automatically satisfied if $U$ is sufficiently small, as Lemma 1.1. of [28] implies that in this case $\left(U \cdot\left(\mathbb{A}_{F}^{\infty}\right)^{\times} \cap t^{-1} D^{\times} t\right) / F^{\times}$is a 2-group. We obtain as a consequence (cf. [28], Cor. 1.2):

Proposition 4.3. Let $B$ a topological A-algebra. The natural morphism $S_{\tau, \psi}(U, A) \otimes_{A} B \rightarrow S_{\tau \otimes_{A} B, \psi \otimes_{A} B}(U, B)$ is an isomorphism of B-modules.

Define a left action of $\left(D \otimes_{F} \mathbb{A}_{F}^{\infty}\right)^{\times}$on the set of functions $D^{\times} \backslash\left(D \otimes_{F}\right.$ $\left.\mathbb{A}_{F}^{\infty}\right)^{\times} \rightarrow W_{\tau}$ by setting $(g f)(x):=f(x g)$ for all $g, x \in\left(D \otimes_{F} \mathbb{A}_{F}^{\infty}\right)^{\times}$. Let $S$ be a set of primes of $F$ containing the ramification set of $D$, the primes above $p$, and the primes $v$ for which $U_{v}$ is not a maximal compact subgroup of $D_{v}^{\times}$. Let

$$
\mathbb{T}_{S, A}^{u n i v}=A\left[T_{v}, S_{v}: v \notin S\right]
$$

be the commutative polynomial $A$-algebra in the indicated indeterminates. For each finite place $v \notin S$, let $\varpi_{v}$ be a fixed uniformizer for $F_{v}$. The space $S_{\tau, \psi}(U, A)$ has a natural action of $\mathbb{T}_{S, A}^{u n i v}$, with $S_{v}$ acting via the double coset $U\left(\varpi_{v}{ }^{\varpi_{v}}\right) U$ and $T_{v}$ via $U\left(\varpi_{v}\right) U([28], 1)$; this action does not depend on the choices of uniformizers. The image of $\mathbb{T}_{S, A}^{u n i v}$ in the ring of $A$-module endomorphisms of $S_{\tau, \psi}(U, A)$ is the Hecke algebra acting on $S_{\tau, \psi}(U, A)$. The isomorphism of Prop. 4.3 is Hecke equivariant. 


\subsection{Systems of Hecke eigenvalues}

Let $R$ be a discrete valuation ring with maximal ideal $\mathfrak{m}_{R}$. If $\mathcal{T}$ is a commutative algebra, a system of eigenvalues of $\mathcal{T}$ with values in $R$ is a set theoretic map $\Omega: \mathcal{T} \rightarrow R$. The reduction of $\Omega$ modulo $\mathfrak{m}_{R}$, denoted $\bar{\Omega}$, is the function obtained by composing $\Omega$ with the natural map $R \rightarrow \frac{R}{\mathfrak{m}_{R}}$. Let $R \mathcal{T}=R \otimes_{\mathbb{Z}} \mathcal{T}$; if $M$ is an $R \mathcal{T}$-module, we say that a system of eigenvalues $\Omega: \mathcal{T} \rightarrow R$ occurs in $M$ if there is a non-zero element $m \in M$ such that $T m=\Omega(T) \cdot m$ for all $T \in \mathcal{T}$. By [2], Prop. 1.2.3, we have:

Lemma 4.5. Let $M$ be an $R \mathcal{T}$-module which is finitely generated over $R$. If $\Omega: \mathcal{T} \rightarrow R$ is a system of eigenvalues of $\mathcal{T}$ occurring in $M$, then $\bar{\Omega}: \mathcal{T} \rightarrow \frac{R}{\mathfrak{m}_{R}}$ is a system of eigenvalues of $\mathcal{T}$ occurring in $M \otimes_{R} \frac{R}{\mathfrak{M}_{R}}$.

Moreover, by [6], Lemme 6.11 (cf. also [2], Prop. 1.2.2):

Lemma 4.6. Let $M$ be an $R \mathcal{T}$-module which is finite and free over $R$. Let $\bar{\Omega}: \mathcal{T} \rightarrow \frac{R}{\mathfrak{m}_{R}}$ be a system of eigenvalues of $\mathcal{T}$ occurring in $M \otimes_{R} \frac{R}{\mathfrak{m}_{R}}$. There exists a finite extension of discrete valuation rings $R^{\prime} / R$ with $\mathfrak{m}_{R^{\prime}} \cap R=\mathfrak{m}_{R}$ and a system of eigenvalues $\Omega^{\prime}: \mathcal{T} \rightarrow R^{\prime}$ of $\mathcal{T}$ occurring in $M \otimes_{R} R^{\prime}$ such that, for all $T \in \mathcal{T}$, we have

$$
\Omega^{\prime}(T)\left(\bmod \mathfrak{m}_{R^{\prime}}\right)=\bar{\Omega}(T) \quad \text { in } \frac{R^{\prime}}{\mathfrak{m}_{R^{\prime}}} .
$$

Let $D, U, \tau, W_{\tau}$ and $\psi$ be as in 4.1, and set $A=\mathcal{O}$. Recall that we are assuming that $\psi$ is compatible with $\left(\tau, W_{\tau}\right), U$ is small enough, and $p$ is odd. Denote by a bar the operation of tensoring over $\mathcal{O}$ with $\mathbb{F}$. From now on we assume fixed a set $S$ of primes of $F$ containing the ramification set of $D$, the primes above $p$, and the primes $v$ for which $U_{v}$ is not a maximal compact subgroup of $D_{v}^{\times}$. The systems of eigenvalues considered below will always be with respect to the Hecke algebra $\mathbb{T}_{S, A^{\prime}}^{u n i v}$ for some topological $\mathcal{O}$-algebra $A^{\prime}$ : we will call such systems Hecke eigensystems.

Lemma 4.7. Fix an $\mathcal{O}$-linear weight $\left(\tau^{\prime}, W_{\tau^{\prime}}\right)$ together with a compatible Hecke character $\psi^{\prime}:\left(\mathbb{A}_{F}^{\infty}\right)^{\times} / F^{\times} \rightarrow \mathcal{O}^{\times}$such that $\bar{\psi}^{\prime}=\bar{\psi}$. Let $\varphi:\left(\bar{\tau}, W_{\bar{\tau}}\right) \rightarrow$ $\left(\bar{\tau}^{\prime}, W_{\bar{\tau}^{\prime}}\right)$ be a non-zero intertwining operator between $\mathbb{F}$-representations of U. Then:

1) $\varphi$ induces a Hecke equivariant $\operatorname{map} \varphi_{*}: S_{\bar{\tau}, \bar{\psi}}(U, \mathbb{F}) \rightarrow S_{\bar{\tau}^{\prime}, \bar{\psi}}(U, \mathbb{F})$;

2) Assume that $\varphi$ is injective. If $\Omega$ is a Hecke eigensystem occurring in $S_{\tau, \psi}(U, \mathcal{O})$, there is a finite extension of $E$ with ring of integers $\mathcal{O}^{\prime}$, 
and there is a Hecke eigensystem $\Omega^{\prime}$ occurring in $S_{\tau^{\prime}, \psi^{\prime}}\left(U, \mathcal{O}^{\prime}\right)$ such that:

$$
\Omega^{\prime}\left(\bmod \mathfrak{m}_{\mathcal{O}^{\prime}}\right)=\Omega\left(\bmod \mathfrak{m}_{\mathcal{O}}\right) \quad \text { in } \frac{\mathcal{O}^{\prime}}{\mathfrak{m}_{\mathcal{O}^{\prime}}}
$$

Proof. For $f \in S_{\bar{\tau}, \bar{\psi}}(U, \mathbb{F})$ set $\varphi_{*}(f):=\varphi \circ f$. As $\bar{\tau}^{\prime}$ and $\bar{\psi}=\bar{\psi}^{\prime}$ are compatible, one sees that $\varphi_{*}(f) \in S_{\bar{\tau}^{\prime}, \bar{\psi}}(U, \mathbb{F})$. If $g, x \in\left(D \otimes_{F} \mathbb{A}_{F}^{\infty}\right)^{\times}$, we have:

$$
\left(g \cdot \varphi_{*}(f)\right)(x)=(\varphi \circ(g \cdot f))(x)=\left(\varphi_{*}(g \cdot f)\right)(x)
$$

so that $\varphi_{*}$ is Hecke-equivariant.

Assume now that $\varphi$ is injective, which also implies the injectivity of $\varphi_{*}$. Let $\Omega$ be a Hecke eigensystem occurring in $S_{\tau, \psi}(U, \mathcal{O})$; by Prop. 4.3, reduction modulo $\mathfrak{m}_{\mathcal{O}}$ induces a Hecke equivariant surjection:

$$
\pi: S_{\tau, \psi}(U, \mathcal{O}) \longrightarrow S_{\bar{\tau}, \bar{\psi}}(U, \mathbb{F})
$$

By Lemma 4.5 , the Hecke eigensystem $\Omega\left(\bmod \mathfrak{m}_{\mathcal{O}}\right)$ occurs in $S_{\bar{\tau}, \bar{\psi}}(U, \mathbb{F})$, and hence in $S_{\bar{\tau}^{\prime}, \bar{\psi}}(U, \mathbb{F})$. Applying Lemma 4.6 to the Hecke equivariant surjection $S_{\tau^{\prime}, \psi^{\prime}}(U, \mathcal{O}) \rightarrow S_{\bar{\tau}^{\prime}, \bar{\psi}}(U, \mathbb{F})$, we deduce the existence of a finite extension of $E$ with ring of integers $\mathcal{O}^{\prime}$, and of a Hecke eigensystem $\Omega^{\prime}: \mathbb{T}_{S, \mathcal{O}^{\prime}} \rightarrow \mathcal{O}^{\prime}$ occurring in $S_{\tau^{\prime}, \psi^{\prime}}(U, \mathcal{O}) \otimes_{\mathcal{O}} \mathcal{O}^{\prime}$ whose reduction modulo $\mathfrak{m}_{\mathcal{O}^{\prime}}$ has value in $\mathbb{F} \subset \frac{\mathcal{O}^{\prime}}{\mathfrak{m}_{\mathcal{O}^{\prime}}}$ and coincide with $\bar{\Omega}$. By Prop. 4.3, $S_{\tau^{\prime}, \psi^{\prime}}(U, \mathcal{O}) \otimes \mathcal{O} \mathcal{O}^{\prime} \simeq S_{\tau^{\prime}, \psi^{\prime}}\left(U, \mathcal{O}^{\prime}\right)$ as Hecke modules, and this concludes the proof.

\subsection{Remarks on regular weights}

For any integer $j$ such that $1 \leq j \leq r$ fix tuples $\mathbf{k}^{(j)}=\left(k_{0}^{(j)}, \ldots, k_{f_{j}-1}^{(j)}\right) \in$ $\mathbb{Z}_{\geq 2}^{f_{j}}$ and $w^{(j)}=\left(w_{0}^{(j)}, \ldots, w_{f_{j}-1}^{(j)}\right) \in \mathbb{Z}^{f_{j}}$. Define the $\mathcal{O}$-module with $G L_{2}(\mathcal{O})$ action:

$$
W_{\left(\mathbf{k}^{(j)}, \mathbf{w}^{(j)}\right)}:=\bigotimes_{i=0}^{f_{j}-1} \operatorname{Sym}^{k_{i}^{(j)}-2} \mathcal{O}^{2} \otimes \operatorname{det}^{w_{i}^{(j)}}
$$

where the tensor products are over $\mathcal{O}$. Letting the group $G L_{2}\left(\mathcal{O}_{F_{\mathfrak{P}_{j}}}\right)$ act on the tensor factor $\operatorname{Sym}^{k_{i}^{(j)}-2} \mathcal{O}^{2} \otimes \operatorname{det}^{w_{i}^{(j)}}$ via the embedding $G L_{2}\left(\mathcal{O}_{F_{\mathfrak{P}_{j}}}\right) \rightarrow$ $G L_{2}(\mathcal{O})$ induced by $\sigma_{i}^{(j)}=\sigma^{i} \circ \sigma_{0}^{(j)}$, the space $W_{\left(\mathbf{k}^{(j)}, \mathbf{w}^{(j)}\right)}$ becomes a representation of $G L_{2}\left(\mathcal{O}_{F_{\mathfrak{P}_{j}}}\right)$. We convene of viewing $G L_{2}\left(\mathcal{O}_{F_{\mathfrak{P}_{j}}}\right)$ as a subgroup of $G L_{2}(\mathcal{O})$ via the embedding $\sigma_{0}^{(j)}$, and we write the $G L_{2}\left(\mathcal{O}_{F_{\mathfrak{P}_{j}}}\right)$-representation 
$W_{\left(\mathbf{k}^{(j)}, \mathbf{w}^{(j)}\right)}$ as:

$$
W_{\left(\mathbf{k}^{(j)}, \mathbf{w}^{(j)}\right)}=\bigotimes_{i=0}^{f_{j}-1}\left(\operatorname{Sym}^{k_{i}^{(j)}-2} \mathcal{O}^{2} \otimes \operatorname{det}^{w_{i}^{(j)}}\right)^{[i]}
$$

where the superscript $[i]$ indicates twisting by the $i$ th power of the Frobenius element $\sigma$.

Denote by $\tau_{\left(\mathbf{k}^{(j)}, \mathbf{w}^{(j)}\right)}$ the action of $G L_{2}\left(\mathcal{O}_{F_{\mathfrak{P}_{j}}}\right)$ on $W_{\left(\mathbf{k}^{(j)}, \mathbf{w}^{(j)}\right)}$ and let $\tau_{(\mathbf{k}, \mathbf{w})}=\bigotimes_{j=1}^{r} \tau_{\left(\mathbf{k}^{(j)}, \mathbf{w}^{(j)}\right)}$, where $\mathbf{k}=\left(\mathbf{k}^{(1)}, \ldots, \mathbf{k}^{(r)}\right)$. We have

$$
\tau_{(\mathbf{k}, \mathbf{w})}: \prod_{j=1}^{r} G L_{2}\left(\mathcal{O}_{F_{\mathfrak{P}_{j}}}\right) \longrightarrow \operatorname{Aut} W_{(\mathbf{k}, \mathbf{w})},
$$

with $W_{(\mathbf{k}, \mathbf{w})}=\bigotimes_{j=1}^{r} W_{\left(\mathbf{k}^{(j)}, \mathbf{w}^{(j)}\right)}($ tensor product over $\mathcal{O})$.

Definition 4.9. We say that $\tau_{(\mathbf{k}, \mathbf{w})}$ is a regular weight if there exists an integer $w$ such that:

$$
k_{i}^{(j)}+2 w_{i}^{(j)}-1=w
$$

for all $j$ and all $i$. In this case, $\tau_{(\mathbf{k}, \mathbf{w})}$ is determined by the weight parameter $(\mathbf{k}, w) \in \mathbb{Z}_{\geq 2}^{g} \times \mathbb{Z}$.

Remark 4.10. We are mostly interested in regular weights. This is because automorphic forms on $D^{\times}$(in the sense of Def. 4.2) having regular weights correspond to classical automorphic representations for $D^{\times}$, cf. [17], 3.1.14, and [28], Lemma 1.3 .

Lemma 4.11. Let $\tau_{(\mathbf{k}, w)}$ be a regular weight, viewed as an $\mathcal{O}$-linear representation of the compact open subgroup $U$ of $\left(D \otimes_{F} \mathbb{A}_{F}^{\infty}\right)^{\times}$. A Hecke character $\psi:\left(\mathbb{A}_{F}^{\infty}\right)^{\times} / F^{\times} \rightarrow \mathcal{O}^{\times}$is compatible with $\tau_{(\mathbf{k}, w)}$ if and only if the following two conditions are satisfied:

1) $\psi(u)=1$ for all $u \in U_{v} \cap \mathcal{O}_{F_{v}}^{\times}$, where $v \in S_{f}$ and $v \nmid p$;

2) $\psi(u)=\left(\operatorname{Nm}_{F_{\mathfrak{P}_{j}} / \mathbb{Q}_{p}}(u)\right)^{1-w}$ for all $u \in \mathcal{O}_{F_{\mathfrak{P}_{j}}}^{\times}$, where $1 \leq j \leq r$.

Proof. The reason for the first condition is straightforward, as the homomorphism $\tau_{(\mathbf{k}, w)}$ factors through $\prod_{j=1}^{r} G L_{2}\left(\mathcal{O}_{F_{\mathfrak{P}_{j}}}\right)$.

Let $j$ be such that $1 \leq j \leq r$ and fix $u \in \mathcal{O}_{F_{\mathfrak{P}_{j}}}^{\times}$; recall that we embed $\mathcal{O}_{F_{\mathfrak{P}_{j}}}$ in $\mathcal{O}$ via $\sigma_{0}^{(j)}$. The matrix $\left({ }^{u}{ }_{u}\right) \in G L_{2}\left(\mathcal{O}_{F_{\mathfrak{P}_{j}}}\right)$ acts on $W_{\left(\mathbf{k}^{(j)}, w\right)}$ as the 
automorphism:

$$
\bigotimes_{i=0}^{f_{j}-1}\left(\sigma^{i}(u)^{k_{i}^{(j)}-2+2 w_{i}^{(j)}} \cdot I d_{i}\right)=\left(\mathrm{Nm}_{F_{\mathfrak{P}_{j}} / \mathbb{Q}_{p}}(u)\right)^{w-1} \cdot I d_{W_{\left(\mathbf{k}^{(j)}, w\right)}}
$$

where $I d_{i}$ denotes the identity map of $\left(\operatorname{Sym}^{k_{i}^{(j)}}-2 \mathcal{O}^{2} \otimes \operatorname{det}^{w_{i}^{(j)}}\right)^{[i]}$. The lemma now follows, as we need to have $\tau_{\mid \mathcal{O}_{F_{\mathfrak{P}_{j}}}^{\times}}(u)=\psi^{-1}(u) \cdot I d_{W_{(\mathbf{k}, w)}}$.

Lemma 4.12. Let $w$ be an even integer. There exists a continuous Hecke character $\psi:\left(\mathbb{A}_{F}^{\infty}\right)^{\times} / F^{\times} \rightarrow \mathbb{Z}_{p}^{\times}$such that:

1) $\psi(u)=1$ for all $u \in \mathcal{O}_{F_{v}}^{\times}$, where $v \in S_{f}$ and $v \nmid p$;

2) $\psi(u)=\left(\mathrm{Nm}_{F_{\mathfrak{P}_{j}} / \mathbb{Q}_{p}}(u)\right)^{w}$ for all $u \in \mathcal{O}_{F_{\mathfrak{P}_{j}}}^{\times}$, where $1 \leq j \leq r$.

Proof. The adèles norm map $\left(\mathbb{A}_{F}^{\infty}\right)^{\times} \rightarrow\left(\mathbb{A}_{\mathbb{Q}}^{\infty}\right)^{\times}$induces a continuous homomorphism Nm : $\left(\mathbb{A}_{F}^{\infty}\right)^{\times} / F^{\times} \rightarrow\left(\mathbb{A}_{\mathbb{Q}}^{\infty}\right)^{\times} / \mathbb{Q}^{\times}$. The decomposition $\left(\mathbb{A}_{\mathbb{Q}}^{\infty}\right)^{\times}=\mathbb{Q}^{\times}$. $\hat{\mathbb{Z}}^{\times}$induces a continuous isomorphism $\beta:\left(\mathbb{A}_{\mathbb{Q}}^{\infty}\right)^{\times} / \mathbb{Q}^{\times} \rightarrow \hat{\mathbb{Z}}^{\times} /\langle-1\rangle$. Moreover, the map $\prod_{l} \mathbb{Z}_{l}^{\times} \rightarrow \mathbb{Z}_{p}^{\times}$defined by sending the tuple $\left(a_{l}\right)_{l} \in \prod_{l} \mathbb{Z}_{l}^{\times}$into $a_{p}^{w} \in \mathbb{Z}_{p}^{\times}$defines a continuous homomorphism $\alpha: \hat{\mathbb{Z}}^{\times} /\langle-1\rangle \rightarrow \mathbb{Z}_{p}^{\times}$since $w$ is even. The composition $\psi:=\alpha \circ \beta \circ \mathrm{Nm}$ is a Hecke character with the desired properties.

Proposition 4.13. Assume $\tau=\tau_{(\mathbf{k}, w)}$ and $\tau^{\prime}=\tau_{\left(\mathbf{k}^{\prime}, w^{\prime}\right)}$ are two regular $\mathcal{O}$ linear weights for automorphic forms on $D$, with $w \equiv w^{\prime}(\bmod p-1)$ and $w$ odd. Assume that $\psi:\left(\mathbb{A}_{F}^{\infty}\right)^{\times} / F^{\times} \rightarrow \mathcal{O}^{\times}$is a Hecke character compatible with $\tau_{(\mathbf{k}, w)}$, and that $\bar{\tau}_{(\mathbf{k}, w)}$ is isomorphic to an $\mathbb{F}$-linear $U$-subrepresentation of $\bar{\tau}_{\left(\mathbf{k}^{\prime}, w^{\prime}\right)}$. Then:

1) There is a Hecke character $\psi^{\prime}:\left(\mathbb{A}_{F}^{\infty}\right)^{\times} / F^{\times} \rightarrow \mathcal{O}^{\times}$which is compatible with $\tau_{\left(\mathbf{k}^{\prime}, w^{\prime}\right)}$ and such that $\bar{\psi}^{\prime}=\bar{\psi}$;

2) For any Hecke eigensystem $\Omega$ occurring in $S_{\tau, \psi}(U, \mathcal{O})$ there is a finite extension of $E$ with ring of integers $\mathcal{O}^{\prime}$, and there is a Hecke eigensystem $\Omega^{\prime}$ occurring in $S_{\tau^{\prime}, \psi^{\prime}}\left(U, \mathcal{O}^{\prime}\right)$ such that $\Omega^{\prime}\left(\bmod \mathfrak{m}_{\mathcal{O}^{\prime}}\right)=\Omega\left(\bmod \mathfrak{m}_{\mathcal{O}}\right)$.

Proof. Since $p>2$, the integer $1-w^{\prime}$ is even. By Lemma 4.12, there exists a Hecke character $\psi^{\prime \prime}:\left(\mathbb{A}_{F}^{\infty}\right)^{\times} / F^{\times} \rightarrow \mathbb{Z}_{p}^{\times} \subset \mathcal{O}^{\times}$such that $\psi^{\prime \prime}(u)=1$ for all $v \in S_{f}$ not lying above $p$ and all $u \in \mathcal{O}_{F_{v}}^{\times}$, and $\psi^{\prime \prime}(u)=\left(\operatorname{Nm}_{F_{\mathfrak{P}_{j}} / \mathbb{Q}_{p}}(u)\right)^{1-w^{\prime}}$ for $u \in \mathcal{O}_{F_{\mathfrak{P}_{j}}}^{\times}$. By Lemma 4.11, $\psi^{\prime \prime}$ is compatible with $\tau_{\left(\mathbf{k}^{\prime}, w^{\prime}\right)}$. Let $\alpha$ denote the reduction modulo $\mathfrak{m}_{\mathcal{O}}$ of the Hecke character $\psi^{-1} \psi^{\prime \prime}$. Since $w \equiv$ 
$w^{\prime}(\bmod p-1)$, by the compatibility of $\psi$ with $\tau_{(\mathbf{k}, w)}$ and by the construction of $\psi^{\prime \prime}$, the continuous character $\alpha$ is trivial on the open subgroup

$$
\prod_{v \nmid p}\left(U_{v} \cap \mathcal{O}_{F_{v}}^{\times}\right) \times \prod_{j=1}^{r} \mathcal{O}_{F_{\mathfrak{P}_{j}}}^{\times}
$$

of $\left(\mathcal{O}_{F} \otimes_{\mathbb{Z}} \hat{\mathbb{Z}}\right)^{\times}$. Therefore $\alpha$ factors through a finite quotient of $\left(\mathbb{A}_{F}^{\infty}\right)^{\times} / F^{\times}$ and the Teichmüller lift $\tilde{\alpha}$ of $\alpha$ is a continuous character $\left(\mathbb{A}_{F}^{\infty}\right)^{\times} / F^{\times} \rightarrow \mathcal{O}^{\times}$. The $\mathcal{O}^{\times}$-valued Hecke character $\psi^{\prime}:=\psi^{\prime \prime} \tilde{\alpha}^{-1}$ is compatible with $\tau_{\left(\mathbf{k}^{\prime}, w^{\prime}\right)}$ and satisfies $\bar{\psi}^{\prime}=\bar{\psi}$, so that the first part of the proposition is proved. The second part follows by applying Lemma 4.7 .

\subsection{Regular weight shiftings}

Set $A=\mathcal{O}$ and let $D, U,\left(\tau, W_{\tau}\right)$, and $\psi$ be as in 4.1. Assume $\tau$ is regular of weight parameter $(\mathbf{k}, w) \in \mathbb{Z}_{\geq 2}^{g} \times \mathbb{Z}$. Recall that we write $\mathbf{k}=\left(\mathbf{k}^{(1)}, \ldots, \mathbf{k}^{(r)}\right)$ with $\mathbf{k}^{(j)}=\left(k_{0}^{(j)}, \ldots, k_{f_{j}-1}^{(j)}\right) \in \mathbb{Z}_{\geq 2}^{f_{j}}$.

The following is an example of a weight shifting result for automorphic forms of regular weight that can be obtained via the operators introduced in 3.5:

Theorem 4.15. Let $\tau$ be a regular $\mathcal{O}$-linear weight with parameters $(\mathbf{k}, w)$ with $w$ odd. Let $f:=\min \left\{f_{1}, \ldots, f_{r}\right\}$ and fix an integer $\beta$ such that $1 \leq \beta \leq$ $f$. For any integers $i, j$ with $1 \leq j \leq r$ and $0 \leq i \leq f_{j-1}$ choose $a_{i}^{(j)} \in\left\{p^{\beta}-\right.$ $\left.1, p^{\beta}+1\right\}$. Set $\mathbf{a}=\left(\mathbf{a}^{(1)}, \ldots, \mathbf{a}^{(r)}\right)$ with $\mathbf{a}^{(j)}=\left(a_{0}^{(j)}, \ldots, a_{f_{j}-1}^{(j)}\right)$, and let $w^{\prime}:=$ $w+\left(p^{\beta}-1\right)$. Assume at least one of the following conditions is satisfied:

1) Let $j$ be any integer such that $1 \leq j \leq r$ and $\beta<f_{j}$. Then for any $i$ with $0 \leq i \leq f_{j}-1$ and $a_{i}^{(j)}=p^{\beta}-1$, we have $2<k_{i}^{(j)} \leq p+1,2 \leq$ $k_{i+f_{j}-\beta}^{(j)} \leq p+1$, and if $i^{\prime} \neq i$ is another integer such that $0 \leq i^{\prime} \leq f_{j}-$ 1 and $a_{i^{\prime}}^{(j)}=p^{\beta}-1$, we also have $i \not \equiv i^{\prime}-\beta\left(\bmod f_{j}\right)$.

Let $j$ be any integer such that $1 \leq j \leq r$ and $\beta=f_{j}$. Then for any $i$ with $0 \leq i \leq f_{j}-1$ and $a_{i}^{(j)}=p^{\beta}-1$, we have $2<k_{i}^{(j)} \leq p+1$.

2) The weight $\mathbf{k}$ satisfies $2<k_{i}^{(j)} \leq p+1$ for all $i, j$.

Let $\psi:\left(\mathbb{A}_{F}^{\infty}\right)^{\times} / F^{\times} \rightarrow \mathcal{O}^{\times}$be a Hecke character compatible with $\tau$. Then if $\Omega$ is a Hecke eigensystem occurring in $S_{\tau, \psi}(U, \mathcal{O})$, there is a finite extension of $E$ with ring of integers $\mathcal{O}^{\prime}$, and there is an $\mathcal{O}^{\prime}$-valued Hecke eigensystem $\Omega^{\prime}$ occurring in regular weight $\left(\mathbf{k}+\mathbf{a}, w^{\prime}\right)$ and with associated Hecke 
character $\psi^{\prime}$ such that:

$$
\Omega^{\prime}\left(\bmod \mathfrak{m}_{\mathcal{O}^{\prime}}\right)=\Omega\left(\bmod \mathfrak{m}_{\mathcal{O}}\right)
$$

The character $\psi^{\prime}$ is compatible with the weight $\left(\mathbf{k}+\mathbf{a}, w^{\prime}\right)$ and it can be chosen so that $\bar{\psi}^{\prime}=\bar{\psi}$.

Proof. Recall that $\tau$ is an $\mathcal{O}$-linear representation $\tau: \prod_{j=1}^{r} G L_{2}\left(\mathcal{O}_{F_{\mathfrak{P}_{j}}}\right) \rightarrow$ Aut $W$ where $W=\bigotimes_{j=1}^{r} W_{j}$ and

$$
W_{j}=\bigotimes_{i=0}^{f_{j}-1}\left(\operatorname{Sym}^{k_{i}^{(j)}-2} \mathcal{O}^{2} \otimes \operatorname{det}^{w_{i}^{(j)}}\right)^{[i]}
$$

By regularity we have $k_{i}^{(j)}+2 w_{i}^{(j)}-1=w$. The group $G L_{2}\left(\mathcal{O}_{F_{\mathfrak{P}_{j}}}\right)$ acts on $W$ via the action on $W_{j}$ induced by the embedding $\sigma_{0}^{(j)}: G L_{2}\left(\mathcal{O}_{F_{\mathfrak{P}_{j}}}\right) \hookrightarrow$ $G L_{2}(\mathcal{O})$. The $\mathbb{F}$-linear representation $\bar{W}_{j}:=W_{j} \otimes_{\mathcal{O}} \mathbb{F}$ of $G L_{2}\left(\mathcal{O}_{F_{\mathfrak{P}_{j}}}\right)$ factors through the reduction map $G L_{2}\left(\mathcal{O}_{F_{\mathfrak{P}_{j}}}\right) \rightarrow G L_{2}\left(\mathbb{F}_{\mathfrak{P}_{j}}\right)$; using the notation introduced in Section 2, we can identify $\bar{W}_{j}$ with the $\mathbb{F}\left[G L_{2}\left(\mathbb{F}_{\mathfrak{P}_{j}}\right)\right]$-module

$$
\bar{W}_{j}=\bigotimes_{i=0}^{f_{j}-1}\left(M_{k_{i}^{(j)}-2} \otimes \operatorname{det}^{w_{i}^{(j)}}\right)^{[i]}
$$

where we see $G L_{2}\left(\mathbb{F}_{\mathfrak{P}_{j}}\right) \hookrightarrow G L_{2}(\mathbb{F})$ via $\bar{\sigma}_{0}^{(j)}$, and the superscript $[i]$ indicates twisting by the $i$ th power of the arithmetic Frobenius of $\operatorname{Gal}\left(\mathbb{F} / \mathbb{F}_{p}\right)$.

For any integer $j$ with $1 \leq j \leq r$, let

$$
\mathcal{T}_{j}=\left\{i: a_{i}^{(j)}=p^{\beta}+1\right\} \text { and } \mathcal{D}_{j}=\left\{i: a_{i}^{(j)}=p^{\beta}-1\right\}
$$

For $i \in \mathcal{T}_{j}$ set $\vartheta_{i}^{(j)}:=\Theta_{f_{j}-\beta}^{[i]}$ if $\beta<f_{j}$ and $\vartheta_{i}^{(j)}:=\Theta^{[i]}$ if $\beta=f_{j}$, where $\Theta_{f_{j}-\beta}^{[i]}$ and $\Theta^{[i]}$ are the generalized Dickson invariants for the group $G L_{2}\left(\mathbb{F}_{\mathfrak{P}_{j}}\right) \simeq$ $G L_{2}\left(\mathbb{F}_{p^{f_{j}}}\right)$ as defined in 3.5.1. For $i \in \mathcal{D}_{j}$ set $\delta_{i}^{(j)}:=D_{f_{j}-\beta}^{[i]}$ if $\beta<f_{j}$ and $\delta_{i}^{(j)}:=D^{[i]}$ if $\beta=f_{j}$, where $D_{f_{j}-\beta}^{[i]}$ and $D^{[i]}$ are the generalized $D$-operators for $G L_{2}\left(\mathbb{F}_{\mathfrak{P}_{j}}\right)$ defined in 3.7.1. Set:

$$
\Lambda_{j}=\left(\bigcirc_{i \in \mathcal{T}_{j}} \vartheta_{i}^{(j)}\right) \circ\left(\bigcirc_{i \in \mathcal{D}_{j}} \delta_{i}^{(j)}\right)
$$

where the symbol $\bigcirc$ denotes composition of functions, and each of the two composition factors is computed by ordering $\mathcal{T}_{j}$ and $\mathcal{D}_{j}$ in the natural way. The operators $\vartheta_{i}^{(j)}$ and $\delta_{i}^{(j)}$ give rise to morphisms of $\mathbb{F}\left[G L_{2}\left(\mathbb{F}_{\mathfrak{P}_{j}}\right)\right]$ modules via the scalar extension $\bar{\sigma}_{0}^{(j)}: \mathbb{F}_{\mathfrak{P}_{j}} \hookrightarrow \mathbb{F}$. We deduce that $\Lambda_{j}$ induces 
a $G L_{2}\left(\mathbb{F}_{\mathfrak{P}_{j}}\right)$-equivariant morphism $\Lambda_{j}: \bar{W}_{j} \rightarrow \bar{W}_{j}^{\prime}$, where $\bar{W}_{j}^{\prime}$ is the $\mathbb{F}\left[G L_{2}\left(\mathbb{F}_{\mathfrak{P}_{j}}\right)\right]$-module:

$$
\begin{aligned}
\bar{W}_{j}^{\prime}:= & \bigotimes_{i \in \mathcal{T}_{j}}\left(M_{k_{i}^{(j)}+\left(p^{\beta}+1\right)-2} \otimes \operatorname{det}^{w_{i}^{(j)}-1}\right)^{[i]} \\
& \otimes \bigotimes_{i \in \mathcal{D}_{j}}\left(M_{k_{i}^{(j)}+\left(p^{\beta}-1\right)-2} \otimes \operatorname{det}^{w_{i}^{(j)}}\right)^{[i]}
\end{aligned}
$$

By 3.5.1, $\bigcirc_{i \in \mathcal{T}_{j}} \vartheta_{i}^{(j)}$ is injective. If $(i)$ is satisfied, the discussion in 3.7.1 implies that $\bigcirc_{i \in \mathcal{D}_{j}} \delta_{i}^{(j)}$ is injective on $\bar{W}_{j}$. Moreover the image of

$$
\bigotimes_{i=0}^{f_{j}-1}\left(X^{k_{i}^{(j)}-2} \otimes 1\right)^{[i]} \in \bar{W}_{j}
$$

under $\bigcirc_{i \in \mathcal{D}_{j}} \delta_{i}^{(j)}$ is of the form $\prod_{i \in \mathcal{D}_{j}}\left(k_{i}^{(j)}-2\right) \cdot u$ for some non-zero $u \in \bar{W}_{j}$. If (ii) holds, $\prod_{i \in \mathcal{D}_{j}}\left(k_{i}^{(j)}-2\right)$ is non-zero in $\mathbb{F}$ and, being $\bar{W}_{j}$ an irreducible representation of $G L_{2}\left(\mathbb{F}_{\mathfrak{P}_{j}}\right)$, we deduce that $\bigcirc_{i \in \mathcal{D}_{j}} \delta_{i}^{(j)}$ is injective on $\bar{W}_{j}$.

We conclude that under assumptions $(i)$ or $(i i)$, all the maps $\Lambda_{j}$ for $1 \leq j \leq r$ are injective. Let $b_{i}^{(j)}=-1$ if $i \in \mathcal{T}_{j}$ and $b_{i}^{(j)}=0$ if $i \in \mathcal{D}_{j}$. Define the $\mathcal{O}\left[G L_{2}\left(\mathcal{O}_{F_{\mathfrak{P}_{j}}}\right)\right]$-module:

$$
W_{j}^{\prime}=\bigotimes_{i=0}^{f_{j}-1}\left(\operatorname{Sym}^{k_{i}^{(j)}+a_{i}^{(j)}-2} \mathcal{O}^{2} \otimes \operatorname{det}^{w_{i}^{(j)}+b_{i}^{(j)}}\right)^{[i]},
$$

so that $W_{j}^{\prime} \otimes_{\mathcal{O}} \mathbb{F}=\bar{W}_{j}^{\prime}$ as $\mathbb{F}$-representations of $G L_{2}\left(\mathcal{O}_{F_{\mathfrak{P}_{j}}}\right)$. Set $W^{\prime}=$ $\bigotimes_{j=1}^{r} W_{j}^{\prime}$ and denote by $\tau^{\prime}$ the action of $U$ on $W^{\prime}$ induced by the projection $U \rightarrow \prod_{j=1}^{r} G L_{2}\left(\mathcal{O}_{F_{\mathfrak{P}_{j}}}\right)$. Let $w^{\prime}=w+\left(p^{\beta}-1\right)$; for all $i$ and $j$ we have $k_{i}^{(j)}+a_{i}^{(j)} \geq k_{i}^{(j)} \geq 2$ and:

$$
\left(k_{i}^{(j)}+a_{i}^{(j)}\right)+2\left(w_{i}^{(j)}+b_{i}^{(j)}\right)-1=w+\left(p^{\beta}-1\right) .
$$

Therefore $\tau^{\prime}$ is a regular weight for automorphic forms on $D$ with parameters $\left(\mathbf{k}+\mathbf{a}, w^{\prime}\right) \in \mathbb{Z}_{\geq 2}^{g} \times \mathbb{Z}$. The injections $\Lambda_{j}$ constructed above allow us to see $\bar{W}=\bigotimes_{j=1}^{r} \bar{W}_{j}$ as an $\mathbb{F}$-linear $U$-subrepresentation of $\bar{W}^{\prime}=\bigotimes_{j=1}^{r} \bar{W}_{j}^{\prime}$. Since $w$ is odd and $w \equiv w^{\prime}(\bmod p-1)$, we can apply Prop. 4.13, which concludes the proof.

Corollary 4.16. Under the assumptions of Th. 4.15, any $\overline{\mathbb{F}}_{p}$-linear continuous Galois representation arising from a Hecke eigenform in $S_{\tau, \psi}(U, \mathcal{O})$, where $\tau$ is a regular weight of parameter $(\mathbf{k}, w)$, also arises from a Hecke 
eigenform in $S_{\tau^{\prime}, \psi^{\prime}}\left(U, \overline{\mathbb{Z}}_{p}\right)$, where $\tau^{\prime}$ is a regular weight of parameter $(\mathbf{k}+$ $\left.\mathbf{a}, w^{\prime}\right)$ and $\psi^{\prime}$ is some $\mathcal{O}^{\times}$-valued Hecke character compatible with $\tau^{\prime}$ and such that $\bar{\psi}^{\prime}=\bar{\psi}$.

Remark 4.17. While the generalized Dickson invariants induce injective maps on the trivial $\mathbb{F}$-representation of $G L_{2}\left(\mathbb{F}_{\mathfrak{P}_{j}}\right)$, the generalized $D$ operators are identically zero on this representation. Starting with an automorphic form whose weight $\left(\mathbf{k}^{(1)}, \ldots, \mathbf{k}^{(r)}\right)$ is such that $\mathbf{k}^{(j)}=\mathbf{2}$ for some $j$, we can then produce weight shiftings using the operators $\Theta_{\alpha}^{[\beta]}$ but we cannot use the operators $D_{\alpha}^{[\beta]}$. On the other side, the study of weight shiftings by $\mathbf{p}-\mathbf{1}$ for such automorphic forms is motivated by the weight part of Serre's modularity conjecture for totally real fields (cf. for example Lemma 4.6.8 of [10]). Results in this directions are obtained by Edixhoven-Khare ([8] 4, Prop. 1), and by C. Sorensen in unpublished work.

\section{Appendix A. - Presentation of the ring $K_{0}\left(S L_{2}\left(\mathbb{F}_{q}\right)\right)$}

Fix a rational prime $p$, a positive integer $g$, and set $q=p^{g}$. Denote by $\mathbb{F}_{q}$ a finite field with $q$ elements and let $\mathfrak{G}$ be the group $S L_{2}\left(\mathbb{F}_{q}\right)$. In this appendix we apply the identities of Th. 2.7 to determine a presentation of the Grothendieck ring of finitely generated $\mathbb{F}_{q}[\mathfrak{G}]$-modules. We treat the case of $\mathfrak{G}=S L_{2}\left(\mathbb{F}_{q}\right)$ instead of $G L_{2}\left(\mathbb{F}_{q}\right)$ only to simplify the computations. The main result is Th. A.6.

Identities in $K_{0}(\mathfrak{G})$. All the representations in this appendix are representations of $\mathfrak{G}$ : for convenience of the reader, we recall the notation set up in Section 2, using Fraktur letters instead or Roman letters to denote representations.

For any $\mathbb{F}_{q}[\mathfrak{G}]$-module $\mathfrak{M}$ and any integer $i$, denote by $\mathfrak{M}^{[i]}$ the $i$ th Frobenius twist of $\mathfrak{M}$. We let $\mathfrak{X}$ be the standard representation of $\mathfrak{G}$ on $\mathbb{F}_{q}^{2}$, and for $k \geq 0$ we set $\mathfrak{M}_{k}:=\operatorname{Sym}^{k} \mathfrak{X}$. Denote by $K_{0}(\mathfrak{G})$ the Grothendieck ring of finitely generated $\mathbb{F}_{q}[\mathfrak{G}]$-modules. We set $\mathfrak{M}_{-1}:=0$ and for any integer $k \leq-2$ we define $\mathfrak{M}_{k}:=-\mathfrak{M}_{-k-2} \in K_{0}(\mathfrak{G})$ ([25]). The following identities hold in $K_{0}(\mathfrak{G})$ for all $k, h \in \mathbb{Z}$ (Th. 2.7):
$\left(\Delta_{g}\right)$
$\mathfrak{M}_{k}+\mathfrak{M}_{-k-2}=0$
$\left(\Sigma_{g}\right)$
$\left(\Pi_{g}\right)$
$\mathfrak{M}_{k}-\mathfrak{M}_{k-(q+1)}=\mathfrak{M}_{k-(q-1)}-\mathfrak{M}_{k-2 q}$
$\left(\Phi_{g}\right)$
$\mathfrak{M}_{k} \mathfrak{M}_{h}=\mathfrak{M}_{k+h}+\mathfrak{M}_{k-1} \mathfrak{M}_{h-1}$
$\mathfrak{M}_{k}=\mathfrak{M}_{k-p} \mathfrak{M}_{1}^{[1]}-\mathfrak{M}_{k-2 p}$. 


\section{Some polynomials}

Lemma A.1. The ring $K_{0}(\mathfrak{G})$ is generated by $\mathfrak{X}$ as a $\mathbb{Z}$-algebra.

Proof. This lemma follows by observing that the Grothendieck ring $K_{0}\left(S L_{2}\right)$ of $\overline{\mathbb{F}}_{p}$-linear algebraic group representations of $S L_{2}$ is generated by the standard representation, together with the fact due to Steinberg that any irreducible representation of $\mathfrak{G}$ is obtained by taking $\mathbb{F}_{q}$-points of an algebraic representation. We present below also another proof, which will be useful later.

The abelian group $K_{0}(\mathfrak{G})$ is freely generated by the $q$ elements $\prod_{i=0}^{g-1} \mathfrak{M}_{k_{i}}^{[i]}$, where $0 \leq k_{i} \leq p-1$ for any $i$. It is therefore enough to show that for all integers $i$ and $k$ such that $0 \leq i \leq g-1$ and $0 \leq k \leq p-1$ we have $\mathfrak{M}_{k}^{[i]} \in \mathbb{Z}[\mathfrak{X}]$. Applying $\left(\Pi_{g}\right)$ we obtain the recursive relations:

$$
\mathfrak{M}_{2}^{[i]}=\left(\mathfrak{X}^{[i]}\right)^{2}-1, \mathfrak{M}_{n}^{[i]}=\mathfrak{X}^{[i]} \cdot \mathfrak{M}_{n-1}^{[i]}-\mathfrak{M}_{n-2}^{[i]} \quad(n>2)
$$

so that $\mathfrak{M}_{k} \in \mathbb{Z}[\mathfrak{X}]$ for all $k \geq 0$, and $K_{0}(\mathfrak{G})=\mathbb{Z}\left[\mathfrak{X}, \mathfrak{X}^{[1]}, \ldots, \mathfrak{X}^{[g-1]}\right]$. Using $\left(\Phi_{g}\right)$ and $\left(\Delta_{g}\right)$ we see that

$$
\mathfrak{X}^{[i+1]}=\mathfrak{M}_{p}^{[i]}-\mathfrak{M}_{p-2}^{[i]}
$$

and we conclude that $\mathfrak{X}^{[1]}, \ldots, \mathfrak{X}^{[g-1]} \in \mathbb{Z}[\mathfrak{X}]$.

Let $X$ be an indeterminate over $\mathbb{Z}$ and define the following families of polynomials of $\mathbb{Z}[X]$ :

$$
\begin{aligned}
& \left\{\begin{array}{l}
m_{0}(X)=1 \\
m_{1}(X)=X \\
m_{n}(X)=X \cdot m_{n-1}(X)-m_{n-2}(X)(n>1) ;
\end{array}\right. \\
& \left\{\begin{array}{l}
f^{[0]}(X)=X \\
f^{[i]}(X)=m_{p}\left(f^{[i-1]}(X)\right)-m_{p-2}\left(f^{[i-1]}(X)\right)=\left(f^{[1]} \circ f^{[i-1]}\right)(X)(i>0) .
\end{array}\right.
\end{aligned}
$$

For $n \geq 0$, the polynomial $m_{n}(X)$ is monic of degree $n$ and $f^{[n]}(X)$ is monic of degree $p^{n}$.

Lemma A.2. For any non-negative integer $i$, we have $f^{[i]}(\mathfrak{X})=\mathfrak{X}^{[i]}$ in $K_{0}(\mathfrak{G})$. 
Proof. By formula (A.1) we have:

$$
m_{n}(\mathfrak{X})=\mathfrak{M}_{n}
$$

in $K_{0}(\mathfrak{G})(n \geq 0)$. If $i=0$ the statement of the lemma is clear, and if $i=1$ it follows from formulae (A.1) and (A.2). Assuming $i \geq 1$ we have:

$$
f^{[i+1]}(\mathfrak{X})=m_{p}\left(f^{[i]}(\mathfrak{X})\right)-m_{p-2}\left(f^{[i]}(\mathfrak{X})\right)=m_{p}\left(\mathfrak{X}^{[i]}\right)-m_{p-2}\left(\mathfrak{X}^{[i]}\right) .
$$

Since taking Frobenius twist is a ring homomorphism of $K_{0}(\mathfrak{G})$, the rightmost term in the above equality is $m_{p}(\mathfrak{X})^{[i]}-m_{p-2}(\mathfrak{X})^{[i]}$. By formula (A.1), the latter is $\mathfrak{M}_{p}^{[i]}-\mathfrak{M}_{p-2}^{[i]}$, which equals $\mathfrak{X}^{[i+1]}$ by (A.2).

Lemma A.3. There is an isomorphism of rings:

$$
\frac{\mathbb{Z}[X]}{(f[g](X)-X)} \simeq K_{0}(\mathfrak{G})
$$

induced by mapping the indeterminate $X$ into the class of the standard representation $\mathfrak{X}$ of $\mathfrak{G}$.

Proof. By Lemma A.1 the ring homomorphism $\pi: \mathbb{Z}[X] \rightarrow K_{0}(\mathfrak{G})$ induced by $X \mapsto \mathfrak{X}$ is surjective. Since $\mathfrak{X}^{[g]}=\mathfrak{X}$, Lemma A.2 implies that $f^{[g]}(X)-$ $X \in \operatorname{ker} \pi$. Since $f^{[g]}(X)-X$ is a monic polynomial of degree $p^{g}$ and $K_{0}(\mathfrak{G})$ is $\mathbb{Z}$-free of rank $p^{g}$, the map $\pi$ induces the desired isomorphism.

We now determine a formula for the polynomial $f(X)$. For an integer $n$, denotes by $\lfloor n\rfloor$ the largest integer not greater than $n$

Lemma A.4. For any non-negative integer $n$ we have:

$$
m_{n}(X)=\sum_{j=0}^{\lfloor n / 2\rfloor}(-1)^{j}\left(\begin{array}{c}
n-j \\
j
\end{array}\right) X^{n-2 j} .
$$

Proof. We induct on $n \geq 0$. Notice the statement is true if $n \leq 2$. Denote by $m_{n}^{\prime}(X)$ the right hand side of the above formula. If $n>2$ we have:

$$
\begin{aligned}
m_{n}(X)= & X m_{n-1}^{\prime}(X)-m_{n-2}^{\prime}(X) \\
= & \sum_{j=0}^{\lfloor(n-1) / 2\rfloor}(-1)^{j}\left(\begin{array}{c}
n-1-j \\
j
\end{array}\right) X^{n-2 j} \\
& -\sum_{j=0}^{\lfloor(n-2) / 2\rfloor}(-1)^{j}\left(\begin{array}{c}
n-2-j \\
j
\end{array}\right) X^{n-2(j+1)} .
\end{aligned}
$$


If $n>2$ is even, $\lfloor(n-1) / 2\rfloor=(n / 2)-1$ and:

$$
\begin{aligned}
m_{n}(X) & =\sum_{j=0}^{(n / 2)-1}(-1)^{j}\left(\begin{array}{c}
n-1-j \\
j
\end{array}\right) X^{n-2 j}+\sum_{j=1}^{n / 2}(-1)^{j}\left(\begin{array}{c}
n-1-j \\
j-1
\end{array}\right) X^{n-2 j} \\
& =X^{n}+\left(\sum_{j=1}^{(n / 2)-1}(-1)^{j}\left(\begin{array}{c}
n-j \\
j
\end{array}\right) X^{n-2 j}\right)+(-1)^{n / 2} \\
& =\sum_{j=0}^{\lfloor n / 2\rfloor}(-1)^{j}\left(\begin{array}{c}
n-j \\
j
\end{array}\right) X^{n-2 j}=m_{n}^{\prime}(X) .
\end{aligned}
$$

If $n>2$ is odd, $\lfloor(n-1) / 2\rfloor=(n-1) / 2,\lfloor(n-2) / 2\rfloor=(n-3) / 2$ and:

$$
\begin{aligned}
m_{n}(X) & =\sum_{j=0}^{(n-1) / 2}(-1)^{j}\left(\begin{array}{c}
n-1-j \\
j
\end{array}\right) X^{n-2 j}+\sum_{j=1}^{(n-1) / 2}(-1)^{j}\left(\begin{array}{c}
n-1-j \\
j-1
\end{array}\right) X^{n-2 j} \\
& =X^{n}+\left(\sum_{j=1}^{(n-1) / 2}(-1)^{j}\left(\begin{array}{c}
n-j \\
j
\end{array}\right) X^{n-2 j}\right) \\
& =\sum_{j=0}^{\lfloor n / 2\rfloor}(-1)^{j}\left(\begin{array}{c}
n-j \\
j
\end{array}\right) X^{n-2 j}=m_{n}^{\prime}(X) .
\end{aligned}
$$

Using Lemma A.4 one can easily check:

Lemma A.5. Let $n \geq 2$ be an integer. Then:

$$
m_{n}(X)-m_{n-2}(X)=\sum_{j=0}^{\lfloor n / 2\rfloor}(-1)^{j} \frac{n}{n-j}\left(\begin{array}{c}
n-j \\
j
\end{array}\right) X^{n-2 j} .
$$

Combining Lemma A.3 with Lemma A.5 we obtain:

Theorem A.6. Let $g$ be a positive integer, $p$ a prime, $q=p^{g}$, and set $\mathfrak{G}=S L_{2}\left(\mathbb{F}_{q}\right)$. The assignment $\mathfrak{X} \mapsto X$ induces an isomorphism of rings:

$$
K_{0}(\mathfrak{G}) \simeq \frac{\mathbb{Z}[X]}{\left(f^{[g]}(X)-X\right)},
$$

where $f^{[g]}(X)=(f \circ \cdots \circ f)(X)$ is the g-fold self-composition of the monic degree $p$ polynomial with integer coefficients:

$$
f(X):=\sum_{j=0}^{\lfloor p / 2\rfloor}(-1)^{j} \frac{p}{p-j}\left(\begin{array}{c}
p-j \\
j
\end{array}\right) X^{p-2 j} .
$$

It was pointed out by $\mathrm{S}$. Bloch that the polynomial $f(X) \in \mathbb{Z}[X]$ is a Dickson polynomial of the first kind (cf. [5]). Similarly to Chebyshev polynomials, Dickson polynomials satisfy a nesting identity with respect to composition, so that one can easily write formulae for their self-iterations. In the case of $f^{[g]}(X)$, such a formula also follows from Serre's relation $\left(\Sigma_{g}\right)$ : 


$$
f^{[g]}(X)=\sum_{j=0}^{\lfloor q / 2\rfloor}(-1)^{j} \frac{q}{q-j}\left(\begin{array}{c}
q-j \\
j
\end{array}\right) X^{q-2 j}
$$

Remark A.7. Dickson polynomials have many special features (cf. [18]). In particular, a conjecture of Schur (1923) states that if $K$ is a number field with ring of integers $\mathcal{O}_{K}$ and $g(X) \in \mathcal{O}_{K}[X]$ is a polynomial of degree larger than one, inducing a permutation of $\mathcal{O}_{K} / \mathfrak{p}$ for infinitely many maximal ideals $\mathfrak{p}$ of $\mathcal{O}_{K}$, then $g(X)$ is a composition of Dickson polynomials and linear polynomials over $K$. Cf. [9], [29], and [19] for a proof of Schur's conjecture.

Notice that $f^{[g]}(X) \equiv X^{q}(\bmod p)$. More generally, we signal the following fact that was pointed out by G. Savin:

Proposition A.8. Let $\mathbf{G}$ be a simply connected semisimple algebraic group defined and split over $\mathbb{F}_{q}$, and denote by $K_{0}(\mathbf{G})$ the Grothendieck ring of $\mathbb{F}_{q}[\mathbf{G}]$-rational modules of finite $\mathbb{F}_{q}$-dimension. If $\mathfrak{M}$ is an element of $K_{0}(\mathbf{G})$ and $i$ is any non-negative integer, we have:

$$
\mathfrak{M}^{[i]} \equiv \mathfrak{M}^{p^{i}}(\bmod p) \text {. }
$$

Proof. Let $\mathbf{T}$ be a maximal torus of $\mathbf{G}$ defined and split over $\mathbb{F}_{q}$, and denote by $\mathcal{X}=\mathcal{X}(\mathbf{T})$ its character group. For any $\lambda \in \mathcal{X}$, denote by $e(\lambda)$ the corresponding basis element of the group ring $\mathbb{Z}[\mathcal{X}]$, so that $e\left(\lambda+\lambda^{\prime}\right)=e(\lambda) e\left(\lambda^{\prime}\right)$ for any characters $\lambda$ and $\lambda^{\prime}$. Fix a G-module $\mathfrak{M}$ and write its formal character as ch $\mathfrak{M}=\sum_{\lambda \in \mathcal{X}} m_{\lambda} \cdot e(\lambda)$, where $m_{\lambda}$ is the dimension of the $\lambda$-isotypic submodule of $\mathfrak{M}$. For a positive integer $i$, the $p^{i}$ th power automorphism of $\overline{\mathbb{F}}_{q}$ induces an action on $\mathbb{Z}[\mathcal{X}]$ by sending a basis element $e(\lambda)$ to $e\left(p^{i} \lambda\right)$, so that:

$$
\operatorname{ch}\left(\mathfrak{M}^{[i]}\right)=\sum_{\lambda \in \mathcal{X}} m_{\lambda} \cdot e(\lambda)^{p^{i}} \equiv\left(\sum_{\lambda \in \mathcal{X}} m_{\lambda} \cdot e(\lambda)\right)^{p^{i}}(\bmod p)
$$

The formal character $\left(\sum_{\lambda \in \mathcal{X}} m_{\lambda} \cdot e(\lambda)\right)^{p^{i}}$ is the element associated to $\mathfrak{M}^{p^{i}}$ by the map ch : $K_{0}(\mathbf{G}) \longrightarrow \mathbb{Z}[\mathcal{X}]$. We have therefore:

$$
\operatorname{ch}\left(\mathfrak{M}^{[i]}\right) \equiv \operatorname{ch}\left(\mathfrak{M}^{p^{i}}\right) \quad(\bmod p) .
$$

Let $\mathcal{W}$ denotes the Weyl group of the pair $(\mathbf{G}, \mathbf{T})$. The proposition now follows using the isomorphism of commutative rings $\mathrm{ch}: K_{0}(\mathbf{G}) \stackrel{\sim}{\longrightarrow} \mathbb{Z}[\mathcal{X}]^{\mathcal{W}}$ (cf. [15] II.5.8). 


\section{Acknowledgements}

The problem of constructing cohomological weight shiftings in the context of the Hasse invariant was conceived and suggested to me by Chandrashekhar Khare, to whom I am deeply indebted. His viewpoint on the problem is the core of [20] and it is fundamental in this paper. I would like to heartily thank C. Khare for his constant, patient, and generous help during the years of my Ph.D. in UCLA, when this paper was conceived. I would like to thank Jean-Pierre Serre, for defining the differential operator $D$ acting on $\mathbb{F}_{q}[X, Y]$, which plays a crucial role in the present paper. I am very grateful to Fred Diamond, who suggested to look into generalizations of the results of [20], and in particular into the existence of cohomological weight shiftings by $(p,-1,0, \ldots, 0)$. I would like to express my gratitude to Claus Sorensen, for sharing with me his unpublished results on cohomological weight shiftings for automorphic forms of trivial weight. I would like to further thank Florian Herzig, for his interest and detailed comments on the results contained in the appendix of the paper, for suggesting how to improve the proof of Lemma A.1, and for sharing his proof of a weakened form of a conjecture appearing in [21]. I would like to thank Spencer Bloch, for signaling that the polynomial appearing in the presentation of $K_{0}\left(S L_{2}\left(\mathbb{F}_{q}\right)\right)$ is a Dickson polynomial. Finally, I would like to thank Don Blasius, Haruzo Hida, Gordan Savin, and Jacques Tilouine for their precious comments and for interesting conversations on the topics considered in this paper, and other topics.

\section{References}

[1] F. Andreatta and E. Z. Goren, Hilbert modular forms: $\bmod p$ and $p$-adic aspects. Memoirs of the American Mathematical Society 819, 2005.

[2] A. Ash and G. Stevens, Cohomology of arithmetic groups and congruences between systems of Hecke eigenvalues. J. Reine Angew. Math., 365 (1986), 192-220.

[3] A. Ash and G. Stevens, Modular forms in characteristic $l$ and special values of their L-functions. Duke Math. J., 53 (1986), 849-868.

[4] K. Buzzard, F. Diamond and F. Jarvis, On Serre's conjecture for mod $l$ Galois representations over totally real fields. Duke Math. J., 155 (2010), 105-161.

[5] L. E. Dickson, The analytic representation of substitutions on a power of a prime number of letters with a discussion of the linear group. Ann. of Math., 11 (1897), 65-120, 161-183. 
[6] P. Deligne and J-P. Serre, Formes modulaires de poids 1. Ann. Scient. Ec. Norm. Sup., 7 (1974), 507-530.

[7] M. Emerton, T. Gee and F. Herzig, Weight cycling and Serre-type conjectures for unitary groups. Duke Math. J., 162 (2013), 1649-1722.

[8] B. Edixhoven and C. Khare, Hasse invariant and group cohomology. Doc. Math., 8 (2003), 43-50.

[9] M. Fried, On a conjecture of Schur. Michigan Math. J., 17 (1970), 4155 .

[10] T. Gee, Automorphic lifts of prescribed types. Math. Ann., 350 (2011), 107-144.

[11] T. Gee, On the weights of mod $p$ Hilbert modular forms. Invent. Math., 184 (2011), 1-46.

[12] E. Z. Goren, Hasse invariants for Hilbert modular varieties. Israel J. Math., 122 (2001), 157-174.

[13] F. Herzig, The weight in a Serre-type conjecture for tame n-dimensional Galois representations. Duke Math. J., 149 (2009), 37-116.

[14] F. Herzig and J. Tilouine, Conjecture de type de Serre et formes compagnons pour GSp4. J. Reine Angew. Math., 676 (2013), 1-32.

[15] J. C. Jantzen, Representations of algebraic groups, Second edition. Mathematical Surveys and Monographs 107, AMS, 2003.

[16] N. M. Katz, p-adic L-functions for CM field. Invent. Math., 49 (1978), 199-297.

[17] M. Kisin, Moduli of finite flat group schemes, and modularity. Ann. of Math., 170 (2009), 1085-1180.

[18] R. Lidl, G. L. Mullen and G. Turnwald, Dickson polynomials. Pitman Monographs and Surveys in Pure and Applied Mathematics 65. Longman Scientific \& Technical, Harlow; copublished in the United States with John Wiley \& Sons, Inc., New York., 1993.

[19] P. Müller, A Weil-bound free proof of Schur's conjecture. Finie Fields Appl., 3 (1997), 25-32.

[20] D. A. Reduzzi, Reduction mod p of cuspidal representations of $G L_{2}\left(\mathbf{F}_{p^{n}}\right)$ and symmetric powers. J. Algebra, 324 (2010), 3507-3531. 
[21] D. A. Reduzzi, An explicit presentation of the Grothendieck ring of finitely generated $\mathbf{F}_{q}\left[S L_{2}\left(\mathbf{F}_{q}\right)\right]$-modules. arXiv:1110.6881 [math.RT], (2011).

[22] H. P. F. Swinnerton-Dyer, On l-adic representations and congruences for coefficients of modular forms. Proceedings of the 1972 Antwerp International Summer School on Modular Forms, Springer Lecture Notes in Mathematics, 350 (1973), 1-55.

[23] J-P. Serre, Congruences et formes modulaires (d'après H.P.F. Swinnerton-Dyer). Esposé 416, Séminaire N. Bourbaki 1971/72, Springer Lecture Notes in Mathematics, 317 (1973), 319-338.

[24] J-P. Serre, Linear representations of finite groups. Springer, New York, 1977.

[25] J-P. Serre, Lettre à Mme Hamer. 2 Juillet 2001.

[26] R. G. Steinberg, Lectures on Chevalley groups. Yale Univ. Press (1968).

[27] R. Taylor, On Galois representations associated to Hilbert modular forms. Invent. Math., 98 (1989), 265-280.

[28] R. Taylor, On the meromorphic continuation of degree two L-functions. Doc. Math. Extra Volume: John Coates' Sixtieth Birthday (2006), 729779 .

[29] G. Turnwald, On Schur's conjecture. J. Austral. Math. Soc. Ser., A 58 (1995), 312-357.

Department of Mathematics, The University of Chicago

5734 S. University, Avenue, Chicago, Illinois 60637, USA

E-mail address: reduzzi@math.uchicago.edu

ReCeived MarCh 8, 2014 